\title{
Local BRST cohomology and Seiberg-Witten maps in noncommutative Yang-Mills theory
}

\author{
Glenn Barnich*, ${ }^{*}$, Friedemann Brandt ${ }^{b}$, and Maxim Grigoriev ${ }^{\dagger, a, c}$ \\ ${ }^{a}$ Physique Théorique et Mathématique, \\ Université Libre de Bruxelles, \\ Campus Plaine C.P. 231, B-1050 Bruxelles, Belgium \\ ${ }^{b}$ Max-Planck-Institute for Mathematics in the Sciences, \\ Inselstraße 22-26, D-04103 Leipzig, Germany \\ ${ }^{c}$ Tamm Theory Department, Lebedev Physical Institute, \\ Leninsky prospect 53, 119991 Moscow, Russia
}

\begin{abstract}
We analyze in detail the recursive construction of the Seiberg-Witten map and give an exhaustive description of its ambiguities. The local BRST cohomology for noncommutative Yang-Mills theory is investigated in the framework of the effective commutative Yang-Mills type theory. In particular, we show how some of the conformal symmetries get obstructed by the noncommutative deformation.
\end{abstract}

${ }^{*}$ Research Associate of the National Fund for Scientific Research (Belgium).

$\dagger$ Postdoctoral Visitor of the National Fund for Scientific Research (Belgium). 


\section{Contents}

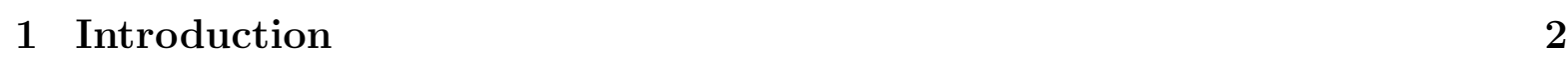

2 Generalities 3

2.1 Reformulation of SW maps in the BV formalism . . . . . . . . . . . . . . . 4

2.2 Local BRST cohomology and SW maps . . . . . . . . . . . . . . . . . . . 6

3 SW maps in noncommutative Yang-Mills theory 6

3.1 Master action and BRST differential . . . . . . . . . . . . . . . . 6

3.2 Existence of SW map from BRST cohomology . . . . . . . . . . . . . . . 7

3.3 Explicit construction of generating functional . . . . . . . . . . . . . . 8

3.4 Recursive construction of SW map . . . . . . . . . . . . . . . . . . . . . 9

3.4.1 Defining equations . . . . . . . . . . . . . . . . . 99 9

3.4.2 Expansion in $\vartheta \ldots \ldots \ldots \ldots$

3.4.3 Expansion in homogeneity in the fields . . . . . . . . . . . . . 12

3.5 Universal SW map . . . . . . . . . . . . . . . . . . . . . . . . . 14

3.6 Ambiguities in the SW map .................. . . . . 14

3.7 Nontriviality of the deformed action. Chern-Simons theorv . . . . . . . . 16

4 Local BRST cohomology of noncommutative $U(N)$ Yang-Mills theory 18

4.1 Basic considerations . . . . . . . . . . . . . . . 18

4.2 Local BRST cohomologv in the effective theorv . . . . . . . . . . . . 18

4.3 Breaking of Poincaré invariance in noncommutative deformation . . . . . 19

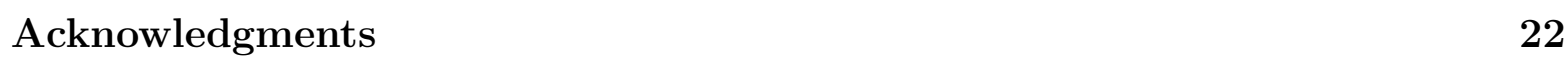

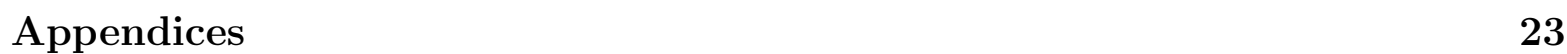

Appendix A: Proof of $(3.7 \mathrm{n}] \ldots \ldots \ldots \ldots \ldots \ldots$

Appendix B: Explicit construction of $\hat{\Xi}$ and $\hat{B}_{0} \ldots \ldots$. . . . . . . . . . . . 25

Appendix C: Proof of proposition $\mathbf{6}$. . . . . . . . . . . . . . . . . . 28

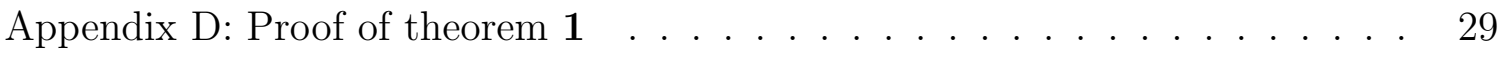

Appendix E: Proof of proposition 7 . . . . . . . . . . . . . . 30

\begin{tabular}{|l|l}
\hline Bibliography & 31
\end{tabular} 


\section{Introduction}

Using arguments from string theory, noncommutative Yang-Mills theory has been shown [1] to be equivalent to a Yang-Mills type theory with standard gauge symmetries and an effective action containing, besides the usual Yang-Mills term, higher dimensional gauge invariant interactions. This equivalence is implemented through a so-called SeibergWitten map (SW map), a redefinition of both the gauge potentials and the parameters of the gauge transformations.

By considering an expansion in some parameter of noncommutativity $\vartheta$, noncommutative Yang-Mills theory can be understood as a consistent deformation of standard Yang-Mills theory in the sense that the action and gauge transformations are deformed simultaneoulsy in such a way that the deformed action is invariant under the deformed gauge transformations. An appropriate framework for analyzing such consistent deformations of gauge theories has been shown [2, 3] to be the antifield-antibracket formalism (see [4, 5, 6, 7] in the Yang-Mills context, 8, 9, 10, 11, 12, for the generic case and 13, 14] for reviews).

By reformulating the question of existence of SW maps in this context, the whole power of the theory of "anti" canonical transformations is available. In the generic case, this leads to an "open" version of the gauge equivalence condition, valid only up to terms vanishing when the equations of motion hold [15. These features have been shown to be crucial for the construction of a SW map for the noncommutative Freedman-Townsend model.

In the case of Yang-Mills and Chern-Simons theory, the relatively simple structure of the gauge algebra allows one to analyze the SW map using antifield independent BRST techniques [16, 17, 18, 19, 20]. Nevertheless, from the point of view of consistent deformations of gauge theories, it is sometimes useful to lift these maps to anticanonical transformations in the field-antifield space, as first discussed in [21, 22].

The first objective of this paper is to improve the explicit recursive construction of the SW map by cohomological methods. The advantage of our solution is the use of explicit expressions for the contracting homotopies adapted respectively to an expansion in the deformation parameter and to an expansion in homogeneity of the fields. These homotopies are based on previous works on the local BRST cohomology of Yang-Mills theory [23, 24]. The methods also allow one to derive the general recursive solution to the SW gauge equivalence condition. In particular, this solution contains additional ambiguities besides those already discussed in [25, 26, 18. We also point out that the ambiguities in the SW map can be understood entirely in the context of the standard Yang-Mills gauge field.

Another aim of this paper is to analyze the local, antifield dependent BRST cohomology groups of noncommutative Yang-Mills theory. These cohomology groups contain the information about the (potential) anomalies, counterterms, and the global symmetries of the model (see e.g. [27] and references therein). In the space of formal power series in the deformation parameter, the analysis can be done in terms of either the noncommutative or the commutative formulation. It turns out to be more convenient to work in the commutative formulation, because one can rely on known results in standard Yang-Mills theory, adapted to the effective theory with higher dimensional interactions. 
Representatives of the cohomology classes in the noncommutative formulation can then be obtained by applying the inverse SW map.

In the next section, we first recall the central equations for the reformulation of SW maps in the antifield formalism. Then we make some general observations on the relation between the BRST cohomology groups of Yang-Mills theory and its noncommutative deformation.

In the first part of section [3, we show in detail that existence of the SW maps follows a priori from known results on the local BRST cohomology of standard YangMills theory [27, 28, 29]. Even though this kind of reasoning does of course not lead to new results for noncommutative Yang-Mills theory, where the existence of SW maps had been proved constructively in the original paper [1], it has been shown to be useful for the existence proof of such maps in more complicated models [15]. In the next part we construct the infinitesimal generating functional for the SW map understood as an anticanonical transformation. The associated evolution equations in the deformation parameter reproduce the original differential equations from [1]. We then give recursive constructions of particular solutions for the SW equations and analyze in detail the ambiguities in the general solution. Finally, we address the question of triviality of the whole noncommutative deformation. In particular, we give the precise argument why noncommutative Yang-Mills theory is a non trivial deformation. Then, we show from cohomological arguments that noncommutative Chern-Simons theory is a trivial deformation of its commutative version, as shown first in [30].

Section 4 is devoted to the local BRST cohomology of noncommutative Yang-Mills theory. Using a Seiberg-Witten map the analysis is done in the commutative formulation. We show that cohomology classes that do not involve the dynamics are unaffected by the noncommutative deformation, while the others can be obstructed. In particular, the breaking of the global Lorentz invariance is discussed in some detail.

Finally, various more technical proofs are given in the appendices.

\section{Generalities}

We assume the space-time manifold to be $\mathbb{R}^{n}$ with coordinates $x^{\mu}, \mu=1, \ldots, n$. Throughout the paper we use notations and conventions from [15]. In particular, the Weyl-Moyal star-product is defined through

$$
f * g(x)=\left.\exp \left(i \wedge_{12}\right) f\left(x_{1}\right) g\left(x_{2}\right)\right|_{x_{1}=x_{2}=x}, \quad \wedge_{12}=\frac{\vartheta}{2} \theta^{\mu \nu} \partial_{\mu}^{x_{1}} \partial_{\nu}^{x_{2}},
$$

for a real, constant, antisymmetric matrix $\theta^{\mu \nu}$. The parameter $\vartheta$ has mass dimension -2 .

A natural space in deformation quantization is the space of formal power series in $\vartheta$ with coefficients in smooth functions. In the context of local field theories, smooth functions are replaced by local functions, i.e., functions that depend on $x^{\mu}$, the fields, and a finite number of their derivatives. More precisely, even though the whole series can depend on an infinite number of derivatives of the fields, each monomial in $\vartheta$ involves only a finite number of them. 
A noncommutative gauge theory is a consistent deformation of its commutative counterpart, in the sense that the action and the gauge transformations are simultaneously deformed in a compatible way. An appropriate framework to describe such consistent deformations is the Batalin-Vilkovisky formalism: the deformations can be described entirely in terms of the master action since it encodes both the gauge invariant action and the gauge transformations. Furthermore, the associated (antifield dependent) BRST cohomology of the undeformed theory controls the deformation 2, 3.

Consider then a noncommutative gauge theory described by the minimal proper solution $\hat{S}\left[\hat{\phi}, \hat{\phi}^{*} ; \vartheta\right]$ of the master equation,

$$
\hat{S}\left[\hat{\phi}, \hat{\phi}^{*} ; \vartheta\right]=\sum_{s=0}^{\infty} \vartheta^{s} S^{(s)}\left[\hat{\phi}, \hat{\phi}^{*}\right]
$$

In particular, the undeformed (commutative) theory is determined by the master action

$$
S^{(0)}\left[\hat{\phi}, \hat{\phi}^{*}\right]=\left.\hat{S}\left[\hat{\phi}, \hat{\phi}^{*} ; \vartheta\right]\right|_{\vartheta=0}
$$

\subsection{Reformulation of SW maps in the BV formalism}

A SW map is by definition a simultaneous field and gauge parameter redefinition such that the gauge structure of the deformed theory is mapped to that of the undeformed one. In the context of the antifield formalism, the existence of a SW map can be expressed in four equivalent ways.

1. There exists an anticanonical field-antifield $\operatorname{transformation}^{1} \hat{\phi}\left[\phi, \phi^{*} ; \vartheta\right], \hat{\phi}^{*}\left[\phi, \phi^{*} ; \vartheta\right]$ such that

$$
\hat{S}\left[\hat{\phi}\left[\phi, \phi^{*} ; \vartheta\right], \hat{\phi}^{*}\left[\phi, \phi^{*} ; \vartheta\right] ; \vartheta\right]=S_{0}^{\mathrm{eff}}[\phi ; \vartheta]+\sum_{r \geq 1} S_{r}^{(0)}\left[\phi, \phi^{*}\right]
$$

where $S_{0}^{\text {eff }}[\hat{\phi} ; 0]=S_{0}^{(0)}[\hat{\phi} ; 0]$ and the subscript denotes the antifield number.

2. There exists a generating functional of "second type" $\mathbf{F}\left[\phi, \hat{\phi}^{*} ; \vartheta\right]$ with

$$
\hat{\phi}^{A}(x)=\frac{\delta^{L} \mathbf{F}}{\delta \hat{\phi}_{A}^{*}(x)}, \quad \phi_{A}^{*}(x)=\frac{\delta^{L} \mathbf{F}}{\delta \hat{\phi}^{A}(x)},
$$

such that

$$
\hat{S}\left[\frac{\delta^{L} \mathbf{F}}{\delta \hat{\phi}^{*}}, \hat{\phi}^{*} ; \vartheta\right]=S_{0}^{\mathrm{eff}}[\phi ; \vartheta]+\sum_{k \geq 1} S_{k}^{(0)}\left[\phi, \frac{\delta^{L} \mathbf{F}}{\delta \phi}\right]
$$

with initial condition $\mathbf{F}=\int d^{n} x \hat{\phi}_{A}^{*} \phi^{A}+O(\vartheta)$.

\footnotetext{
${ }^{1}$ Only anticanonical transformation that reduce to the identity to order 0 in the deformation parameter are considered here. Invertibility of these transformations in the space of formal power series is then guaranteed.
} 
3. There exists a functional $\hat{\Xi}\left[\hat{\phi}, \hat{\phi}_{*} ; \vartheta\right]$ such that

$$
\frac{\partial \hat{S}}{\partial \vartheta}=\hat{B}_{0}+(\hat{S}, \hat{\Xi})
$$

holds with $\hat{B}_{0}[\hat{\phi} ; \vartheta]$. The field-antifield redefinition of formulation 1 can then be constructed as the solution to the differential equations

$$
\begin{aligned}
\frac{\partial \hat{\phi}^{A}}{\partial \vartheta} & =\left(\hat{\Xi}, \hat{\phi}^{A}\right), \\
\frac{\partial \hat{\phi}_{A}^{*}}{\partial \vartheta} & =\left(\hat{\Xi}, \hat{\phi}_{A}^{*}\right)
\end{aligned}
$$

and $\hat{B}_{0}[\hat{\phi} ; \vartheta]=\left(\partial S_{0}^{\text {eff }} / \partial \vartheta\right)[\phi[\hat{\phi} ; \vartheta] ; \vartheta]$. Formally, this solution can be written as

$$
\begin{aligned}
& \hat{\phi}^{A}(x)=\left[P \exp \int_{0}^{\vartheta} d \vartheta^{\prime}\left(\Xi\left(\vartheta^{\prime}\right), \cdot\right)\right] \phi^{A}(x), \\
& \hat{\phi}_{A}^{*}(x)=\left[P \exp \int_{0}^{\vartheta} d \vartheta^{\prime}\left(\Xi\left(\vartheta^{\prime}\right), \cdot\right)\right] \phi_{A}^{*}(x),
\end{aligned}
$$

where $\Xi\left[\phi, \phi^{*} ; \vartheta\right]$ is the same function of $\phi, \phi^{*} ; \vartheta$ as $\hat{\Xi}\left[\hat{\phi}, \hat{\phi}^{*} ; \vartheta\right]$ is of $\hat{\phi}, \hat{\phi}^{*} ; \vartheta$.

4. The deformed and undeformed theories are weakly gauge equivalent in the following sense. Let $\hat{L}_{0}[\hat{\varphi} ; \vartheta]$ be a Lagrangian of the deformed theory and $L_{0}^{\text {eff }}[\varphi ; \vartheta]=$ $\hat{L}_{0}[f[\varphi ; \vartheta]]$ be the respective effective Lagrangian. In the case of an irreducible gauge theory, there exists a simultaneous redefinition of the original gauge fields $\hat{\varphi}^{i}=f^{i}[\varphi ; \vartheta]$ and the parameters ${ }^{2} \hat{\epsilon}^{\alpha}=g_{\beta}^{\alpha}[\varphi ; \vartheta]\left(\epsilon^{\beta}\right)$ of the irreducible generating set of nontrivial gauge transformations $\hat{R}_{\alpha}^{i}[\hat{\phi} ; \vartheta]\left(\hat{\epsilon}^{\alpha}\right)$ such that

$$
\left.\left(\hat{\delta}_{\hat{\epsilon}} \hat{\varphi}^{i}\right)\right|_{\hat{\varphi}=f, \hat{\epsilon}=g} \approx \delta_{\epsilon} f^{i},
$$

where $\approx$ means terms that vanish when the equations of motions associated to $S_{0}^{\text {eff }}=\int d^{n} x L_{0}^{\text {eff }}[\varphi ; \vartheta]$ hold. The operators $\hat{\delta}_{\hat{\epsilon}}, \delta_{\epsilon}$ are given by

$$
\begin{aligned}
& \hat{\delta}_{\hat{\epsilon}}=\sum_{k=0} \partial_{\mu_{1}} \ldots \partial_{\mu_{k}}\left(\hat{R}_{\alpha}^{i}[\hat{\varphi} ; \vartheta]\left(\hat{\epsilon}^{\alpha}\right)\right) \frac{\partial}{\partial\left(\partial_{\mu_{1}} \ldots \partial_{\mu_{k}} \hat{\varphi}^{i}\right)}, \\
& \delta_{\epsilon}=\sum_{k=0} \partial_{\mu_{1}} \ldots \partial_{\mu_{k}}\left(R_{\alpha}^{i}[\varphi]\left(\epsilon^{\alpha}\right)\right) \frac{\partial}{\partial\left(\partial_{\mu_{1}} \ldots \partial_{\mu_{k}} \varphi^{i}\right)},
\end{aligned}
$$

with $R_{\alpha}^{i}[\varphi]\left(\epsilon^{\alpha}\right)$ being the associated irreducible generating set of nontrivial gauge transformations of the undeformed theory.

In what follows, by effective theory we mean the commutative theory described by the Lagrangian $L_{0}^{\text {eff }}[\varphi ; \vartheta]$ or, equivalently, by the solution of the master equation given by the right hand side of (2.4).

\footnotetext{
${ }^{2} \mathrm{~A}$ square bracket means a local dependence on the fields and their derivatives, while the round bracket means that this dependence is linear and homogeneous.
} 


\subsection{Local BRST cohomology and SW maps}

Let $\hat{s}=(\hat{S}, \cdot)$ be the BRST differential of a noncommutative theory admitting a SW map. In the space of formal power series in the deformation parameter with coefficients in local functions or local functionals, the BRST cohomology groups $H(\hat{s})$ are isomorphic to the BRST cohomology groups of the associated effective theory because these two theories are related by an anticanonical field-antifield redefinition. Furthermore, these cohomology groups are included in the associated cohomology groups of the undeformed commutative theory, evaluated in the spaces of formal power series in $\vartheta$.

The BRST differential $s^{\text {eff }}$ of the effective theory can be expanded according to the antifield number as $s^{\text {eff }}=\delta^{\text {eff }}+\gamma+s_{1}+\ldots$ The Koszul-Tate differential $\delta^{\text {eff }}$ lowers the antifield number by 1 and is associated to the equations of motion of $S_{0}^{\text {eff }}[\varphi ; \vartheta]$, while $\gamma, s_{1}, \ldots$ of antifield number $0,1, \ldots$ are identical to the corresponding operators of the undeformed theory.

In the Yang-Mills case that we are interested in here, $\gamma$ is a differential and the operators $s_{1}, \ldots$ all vanish. Hence, the difference between the local BRST cohomology groups of noncommutative Yang-Mills theory and its commutative counterpart is due only to the dynamics encoded in $\delta^{\text {eff }}$ respectively $\delta$.

\section{SW maps in noncommutative Yang-Mills theory}

In the first subsection, we give the master action for noncommutative Yang-Mills theory and the associated BRST differential involving the antifields. We assume that fields take values in $u(N)$ or in some associative matrix algebra $\mathcal{U}$. For simplicity we limit ourselves to pure Yang-Mills theories. The inclusion of matter fields is straightforward along the lines of 31 .

\subsection{Master action and BRST differential}

The minimal (not necessarily proper) solution of the master equation for noncommutative Yang-Mills theory is given by

$$
\hat{S}=\int d^{n} x\left(-\frac{1}{4 \kappa^{2}} \operatorname{Tr}\left(\hat{F}^{\mu \nu} * \hat{F}_{\mu \nu}\right)+\hat{A}_{A}^{* \mu} *\left(\hat{D}_{\mu} \hat{C}\right)^{A}+\hat{C}_{A}^{*} *(\hat{C} * \hat{C})^{A}\right),
$$

where $\hat{A}_{\mu}=\hat{A}_{\mu}^{A} T_{A}$ and $\hat{C}=\hat{C}^{A} T_{A}$ are either $u(N)$ or $\mathcal{U}$-valued gauge fields and ghost fields, $\hat{A}_{A}^{* \mu}$ and $\hat{C}_{A}^{*}$ are the antifields conjugate to $\hat{A}_{\mu}^{A}$ and $\hat{C}^{A}$, and

$$
\hat{F}_{\mu \nu}=\partial_{\mu} \hat{A}_{\nu}-\partial_{\nu} \hat{A}_{\mu}+\left[\hat{A}_{\mu}^{*}, \hat{A}_{\nu}\right], \quad \hat{D}_{\mu} \hat{C}=\partial_{\mu} \hat{C}+\left[\hat{A}_{\mu}^{*}, \hat{C}\right],
$$

with $[*]$ the graded star-commutator,

$$
[A * B]=A * B-(-1)^{|A||B|} B * A .
$$

The BRST differential $\hat{s}$ for the noncommutative model is defined in the standard way as canonically generated by the associated master action:

$$
\hat{s} \cdot=(\hat{S}, \cdot) \text {. }
$$


It is useful to represent $\hat{s}$ as the sum $\hat{s}=\hat{\gamma}+\hat{\delta}$, where $\hat{\gamma}$ is the part of the BRST differential with antifield number 0 and $\hat{\delta}$ is the Koszul-Tate part (see e.g. 13]). The differentials $\hat{\gamma}$ and $\hat{\delta}$ act on the gauge fields, ghost fields, and antifields as follows:

$$
\begin{aligned}
& \hat{\gamma} \hat{A}_{\mu}=\hat{D}_{\mu} \hat{C}, \quad \hat{\gamma} \hat{C}=-\hat{C} * \hat{C}, \\
& \hat{\delta} \hat{A}_{\mu}=0, \quad \hat{\delta} \hat{C}=0, \\
& \hat{\gamma} \hat{A}_{A}^{* \mu}=-\left(T_{A} \hat{C}\right)^{B} * \hat{A}_{B}^{* \mu}-\hat{A}_{B}^{* \mu} *\left(\hat{C} T_{A}\right)^{B}, \\
& \hat{\gamma} \hat{C}_{A}^{*}=-\left(T_{A} \hat{C}\right)^{B} * \hat{C}_{B}^{*}+\hat{C}_{B}^{*} *\left(\hat{C} T_{A}\right)^{B}, \\
& \hat{\delta} \hat{A}_{A}^{* \mu}=\frac{1}{\kappa^{2}} \operatorname{Tr}\left(T_{A} \hat{D}_{\nu} \hat{F}^{\nu \mu}\right), \\
& \hat{\delta} \hat{C}_{A}^{*}=-\partial_{\mu} \hat{A}_{A}^{* \mu}-\left(T_{A} \hat{A}_{\mu}\right)^{B} * \hat{A}_{B}^{* \mu}+\hat{A}_{B}^{* \mu} *\left(\hat{A}_{\mu} T_{A}\right)^{B},
\end{aligned}
$$

where $T_{A} \hat{C}=\left(T_{A} \hat{C}\right)^{B} T_{B}$. The BRST differential $\hat{s}$ can be expanded in $\vartheta$,

In the next subsection, we show that existence of the SW map follows directly from standard results on the BRST cohomology of the commutative Yang-Mills theory.

\subsection{Existence of SW map from BRST cohomology}

As discussed in [15, existence would be direct if there were no antifield dependent cohomology, but even in the $u(N)$ case there is in fact antifield dependent cohomology because of the $\mathrm{U}(1)$ factor. We show that the noncommutative deformation does not involve this cohomology, which completes the previous arguments.

Assume we have constructed a SW map $\hat{\phi}^{k}\left[\phi, \phi^{*} ; \vartheta\right], \hat{\phi}^{* k}\left[\phi, \phi^{*} ; \vartheta\right]$ to order $k$ in $\vartheta$. This means that (2.4) holds up to terms of order $k+1$ and higher:

$$
\begin{aligned}
\hat{S}\left[\hat{\phi}^{k}\left[\phi, \phi^{*} ; \vartheta\right], \hat{\phi}^{* k}\left[\phi, \phi^{*} ; \vartheta\right] ; \vartheta\right]= & \\
& =\sum_{l=0}^{k} \vartheta^{l} S_{0}^{\mathrm{eff}(l)}[A]+\sum_{r \geq 1} S_{r}^{(0)}\left[\phi, \phi^{*}\right]+\vartheta^{k+1} \tilde{S}^{(k+1)}\left[\phi, \phi^{*}\right]+O(k+2),
\end{aligned}
$$

with $\left(S^{(0)}, S_{0}^{\text {eff }(l)}[A]\right)=0$ and $\hat{\phi}^{k}, \hat{\phi}^{* k}$ related to $\phi, \phi^{*}$ through an anticanonical transformation. The master equation $(\hat{S}, \hat{S})=0$ then implies

$$
\left(S^{(0)}, \tilde{S}^{(k+1)}\right)=0 .
$$

Suppose that the cocycle $\tilde{S}^{(k+1)}$ belongs to a subspace $\mathfrak{S}$, where the representatives of the cohomology of $s^{(0)}$ can be chosen to be antifield independent, so that

$$
\left(S^{(0)}, \tilde{S}^{(k+1)}\right)=0 \quad \Longrightarrow \quad \tilde{S}^{(k+1)}=S_{0}^{\mathrm{eff}(k+1)}[A]+\left(S^{(0)}, \tilde{\Xi}^{(k+1)}\right) .
$$

Then, the SW map can be constructed as a succession of anticanonical transformations. Indeed, if we define

$$
\begin{aligned}
\hat{\phi}^{k+1}\left[\phi, \phi^{*} ; \vartheta\right] & =\exp \left(\vartheta^{k+1}\left(\tilde{\Xi}^{(k+1)}, \cdot\right)\right) \hat{\phi}^{k}, \\
\hat{\phi}^{* k+1}\left[\phi, \phi^{*} ; \vartheta\right] & =\exp \left(\vartheta^{k+1}\left(\tilde{\Xi}^{(k+1)}, \cdot\right)\right) \hat{\phi}^{* k},
\end{aligned}
$$


the action $\hat{S}\left[\hat{\phi}^{k+1}, \hat{\phi}^{* k+1} ; \vartheta\right]$ satisfies (3.5) with $k+1$ in place of $k$. For $k=0$, Eq. (3.5) obviously holds with $\hat{\phi}^{0}=\phi, \hat{\phi}^{* 0}=\phi^{*}$ the identity map and $S_{0}^{\text {eff }}{ }^{(0)}[A]$ the standard commutative Yang-Mills action.

In our case, the subspace $\mathfrak{S}$ can be taken to be the space of local functionals depending at most linearly on antifields and depending on the ghosts only via their derivatives when written in terms of undifferentiated antifields. We show in appendix $\mathbf{A}$ that (i) equation (3.7) indeed holds if $\tilde{S}^{(k+1)} \in \mathfrak{S}$ and (ii) that $\tilde{\Xi}^{(k+1)}$ can be chosen in $\mathfrak{S}$. In this case $\tilde{S}^{(k+2)}$ can also be chosen in $\mathfrak{S}$ (through integrations by parts) since all terms of $\hat{S}$ of first and higher order belong to $\mathfrak{S}$, and terms in $\hat{\phi}^{k+1}\left[\phi, \phi^{*} ; \vartheta\right], \hat{\phi}^{* k+1}\left[\phi, \phi^{*} ; \vartheta\right]$ of first and higher order are at most linear in antifields and depend only on differentiated ghosts if $\tilde{\Xi}^{(l)}, l=1 \ldots, k+1$ belong to $\mathfrak{S}$, which completes the proof.

\subsection{Explicit construction of generating functional}

In this subsection, we give a constructive approach to the differential equations of Seiberg and Witten, whose integration provides the map that establishes the equivalence of the gauge structure of the noncommutative and the commutative theories. The differential equations appear here as those for an anticanonical transformation, more precisely a Hamiltonian evolution equation with time replaced by $\vartheta$, the parameter of noncommutativity. Because this generating functional contains the evolution parameter $\vartheta$, the formal solution is obtained by the standard path-ordered exponential associated to time dependent anticanonical transformation.

The generating functional $\hat{\Xi}$ defined by (2.7)

$$
\frac{\partial \hat{S}}{\partial \vartheta}=\hat{B}_{0}+\hat{s} \hat{\Xi}
$$

can be constructed by using an appropriate contracting homotopy. In order to do so, one decomposes the functionals according to the antifield number and expands in homogeneity in the fields. In this case the relevant differential controling the construction is $\hat{\gamma}^{[0]}$ which acts on the fields and antifields simply according to

$$
\hat{\gamma}^{[0]} \hat{A}_{\mu}=\partial_{\mu} \hat{C}, \quad \hat{\gamma}^{[0]} \hat{C}=\hat{\gamma}^{[0]} \hat{A}_{A}^{* \mu}=\hat{\gamma}^{[0]} \hat{C}_{A}^{*}=0 .
$$

The decomposition of $\hat{\gamma}$ then reads

$$
\hat{\gamma}=\hat{\gamma}^{[0]}+\hat{\gamma}^{[1]}
$$

where $\hat{\gamma}^{[1]}$ contains the quadratic terms of the $\hat{\gamma}$-transformations. Details are given in appendix $\mathbf{B}$ and we present here only the final results:

$$
\begin{aligned}
& \hat{\Xi}=\frac{i \theta^{\alpha \beta}}{4} \int d^{n} x\left(-\hat{A}_{A}^{* \mu}\left\{\hat{F}_{\alpha \mu}+\partial_{\alpha} \hat{A}_{\mu}^{*}, \hat{A}_{\beta}\right\}^{A}+\hat{C}_{A}^{*}\left\{\hat{A}_{\alpha}^{*}, \partial_{\beta} \hat{C}\right\}^{A}\right), \\
& \hat{B}_{0}=\frac{i \theta^{\alpha \beta}}{\kappa^{2}} \int d^{n} x \operatorname{Tr}\left(\frac{1}{8} \hat{F}_{\alpha \beta} \hat{F}_{\mu \nu} * \hat{F}^{\mu \nu}-\frac{1}{2} \hat{F}_{\alpha \mu} * \hat{F}_{\beta \nu} \hat{F}^{\mu \nu}\right) .
\end{aligned}
$$


The associated differential equations (2.8) and (2.9) for $\hat{A}_{\mu}$ and $\hat{C}$ are the ones from [1]:

$$
\begin{aligned}
\frac{\partial \hat{A}_{\mu}}{\partial \vartheta} & =\left(\hat{\Xi}, \hat{A}_{\mu}\right)=-\frac{i \theta^{\alpha \beta}}{4}\left\{\hat{A}_{\alpha}, \hat{F}_{\beta \mu}+\partial_{\beta} \hat{A}_{\mu}\right\}, \\
\frac{\partial \hat{C}}{\partial \vartheta} & =(\hat{\Xi}, \hat{C})=\frac{i \theta^{\alpha \beta}}{4}\left\{\partial_{\alpha} \hat{C}^{*}, \hat{A}_{\beta}\right\} .
\end{aligned}
$$

Notice also that equation (3.13) provides directly the effective action to first order in $\vartheta$ :

$$
\begin{aligned}
& S^{\mathrm{eff}}[A ; \vartheta]= \\
& =-\frac{1}{4 \kappa^{2}} \int d^{n} x \operatorname{Tr}\left(F_{\mu \nu} F^{\mu \nu}+\frac{i \vartheta \theta^{\alpha \beta}}{2}\left(-F_{\alpha \beta} F_{\mu \nu} F^{\mu \nu}+4 F_{\alpha \mu} F_{\beta \nu} F^{\mu \nu}\right)\right)+O\left(\vartheta^{2}\right) .
\end{aligned}
$$

\subsection{Recursive construction of SW map}

In this subsection we construct recursive solutions for the SW map. We use the standard technique of homological perturbation theory based either on an expansion in the deformation parameter or an expansion in homogeneity in the fields. In the former case the appropriate coboundary operator is $\bar{\gamma}=\gamma+[C, \cdot]$ while in the latter it is $\gamma^{[0]}$.

\subsubsection{Defining equations}

Linearity in antifields of the generating functional $\hat{\Xi}$ implies that the generating functional $\mathbf{F}$ of second type can also be chosen linear in antifields,

$$
\mathbf{F}=\int d^{n} x\left(\hat{A}_{A}^{* \mu} f_{\mu}^{A}+\hat{C}_{A}^{*} h^{A}\right)
$$

where $f_{\mu}^{A}=f_{\mu}^{A}[A ; \vartheta]$ and $h^{A}=h^{A}[A ; \vartheta](C)$. Then the defining equation (2.6) reduces to

$$
\begin{array}{r}
-\frac{1}{4 \kappa^{2}} \int d^{n} x \operatorname{Tr} \hat{F}_{\mu \nu} \hat{F}^{\mu \nu}[f ; \vartheta]=\int d^{n} x L_{0}^{\mathrm{eff}}[A ; \vartheta], \\
\left(\partial_{\mu} h+\left[f_{\mu}^{*}, h\right]\right)^{A}=\gamma f_{\mu}^{A}, \\
\frac{1}{2}\left[h^{*}, h\right]^{A}+\gamma h^{A}=0,
\end{array}
$$

where $f_{\mu}=f_{\mu}^{A}[A ; \vartheta] T_{A}$ and $h=h^{A}[A, C ; \vartheta] T_{A}$ and $\gamma$ is the gauge part of the BRST differential of the commutative theory:

$$
\begin{aligned}
\gamma f[A, C]=\sum_{k=0} \partial_{\rho_{1}} \ldots \partial_{\rho_{k}}\left(D_{\mu} C\right)^{B} & \frac{\partial^{L} f[A, C]}{\partial\left(\partial_{\rho_{1}} \ldots \partial_{\rho_{k}} A_{\mu}^{B}\right)} \\
& -\sum_{k=0} \frac{1}{2} \partial_{\rho_{1}} \ldots \partial_{\rho_{k}}\left(f_{D E}{ }^{B} C^{D} C^{E}\right) \frac{\partial^{L} f[A, C]}{\partial\left(\partial_{\rho_{1}} \ldots \partial_{\rho_{k}} C^{B}\right)}
\end{aligned}
$$


where $f_{D E}{ }^{B}$ are the structure constants of the Lie algebra associated to the underlying matrix algebra,

$$
\left[T_{A}, T_{B}\right]=f_{A B}^{C} T_{C}
$$

Notice that for a general generating functional $\mathbf{F}$ that is not linear in the antifields, equations (3.18) and (3.19) may contain equation of motion terms [15].

Equation (3.18) is the SW equation (3.3) of [1] under the form

$$
\hat{\delta}_{\hat{\lambda}} \hat{A}=\delta_{\lambda} \hat{A},
$$

with the identifications $\hat{A}_{\mu} \leftrightarrow f_{\mu}, \hat{\lambda} \leftrightarrow h$ and $\lambda \leftrightarrow C$. We are going to solve the BRST version (3.19) of the integrability condition before solving the SW equation (3.18), because it contains as unknown functions only the noncommutative gauge parameter $h$ as a function of $\theta^{\mu \nu}, C^{A}, A_{\mu}^{A}$, and their derivatives.

\subsubsection{Expansion in $\vartheta$}

Assume first that $h^{k}=\sum_{l=0}^{k} \vartheta^{l} h^{(l)}$ solves (3.19) up to order $k+1$ in the deformation parameter $\vartheta$. This means that

$$
\frac{1}{2}\left[h^{k} * h^{k}\right]+\gamma h^{k}=\vartheta^{k+1} r^{(k+1)}+O(k+2),
$$

where in the right hand side we have explicitly written the term of order $k+1$. Applying $\gamma+\left[h^{k}, \cdot\right]$ to this equation the left hand side vanishes identically. The consistency condition

$$
\left(\gamma+\left[h^{k}, \cdot\right]\right)\left(\vartheta^{k+1} r^{(k+1)}+O(k+2)\right)=0
$$

implies to lowest order

$$
(\gamma+[C, \cdot]) r^{(k+1)}=0 .
$$

Thus the operator $\bar{\gamma}=\gamma+[C, \cdot]$ is the natural operator for recursively solving (3.19) and also $(3.18)^{3}$. In particular it is nilpotent, $\bar{\gamma}^{2}=0$, when acting on matrix algebra-valued local functions. Note that unlike the ordinary BRST differential $\gamma, \bar{\gamma}$ doesn't commute with $\partial_{\mu}$. Instead, $\bar{\gamma}$ commutes with the covariant derivative: $\left[\bar{\gamma}, D_{\mu}\right]=0$.

To proceed to the next order one has to show that the cocycle $r^{(k+1)}$ is a coboundary of $\bar{\gamma}$. This can be achieved by using $\rho$ given explicitly by (A.9) which is a contracting homotopy for $\bar{\gamma}$. Indeed, one can check that

$$
\{\bar{\gamma}, \rho\} f(y, z, \chi, C)=f(y, z, \chi, C)-f(0,0, \chi, C),
$$

where $f$ is an algebra-valued local function and the following independent coordinates are introduced in the space of fields and their derivatives (see appendix $\mathbf{A}$ for details):

$$
\begin{aligned}
& \left\{y^{\alpha}\right\}=\left\{\partial_{\left(\nu_{1}\right.} \ldots \partial_{\nu_{l}} A_{\mu)}^{A}\right\}, \quad\left\{z^{\alpha}\right\}=\left\{\partial_{\left(\nu_{1}\right.} \ldots \partial_{\nu_{l}} D_{\mu)} C^{A}\right\}, \\
& \left\{\chi_{\Delta}^{A}\right\}=\left\{D_{\left(\nu_{1}\right.} \ldots D_{\nu_{l}} F_{\mu) \lambda}^{A}\right\}, \quad\left\{C^{A}\right\} .
\end{aligned}
$$

\footnotetext{
${ }^{3}$ In this context the relevance of this operator was first observed in [17] (see also [19).
} 
Proposition 1. A particular recursive solution $h=\sum_{k=0} \vartheta^{k} h^{(k)}$ to equation (3.19) with $h^{(0)}=C$ is given by

$$
h^{(k+1)}=-\rho\left(\sum_{i+j+l=k+1} \frac{1}{2}\left[h^{(i)}, h^{(j)}\right]^{(l)}\right), \quad 0 \leq i, j \leq k, \quad 0 \leq l \leq k+1, \quad k \geq 0,
$$

where we have expanded the *-commutator with respect to powers in $\vartheta$ :

$$
\left[a^{*}, b\right]=\sum_{l=0}^{\infty} \vartheta^{l}\left[a^{*}, b\right]^{l}=[a, b]+\frac{i \vartheta \theta^{\alpha \beta}}{2}\left\{\partial_{\alpha} a, \partial_{\beta} b\right\}+\cdots .
$$

Proof. At zeroth order in $\vartheta$ Eq. (3.19) is satisfied by $h^{0}=C$. Assume that we have constructed $h^{k}=\sum_{l=0}^{k} \vartheta^{l} h^{(l)}$ such that $h^{k}$ solves (3.19) up to order $k+1$ with $h^{(l)}$ independent on undifferentiated ghosts for $1 \leq l \leq k$. At order $k+1$ in $\vartheta$ Eq. (3.19) requires

$$
-\bar{\gamma} h^{(k+1)}=\frac{1}{2} \sum_{i+j+l=k+1}\left[h^{(i)}, h^{(j)}\right]^{(l)}, \quad 0 \leq i, j \leq k, \quad 0 \leq l \leq k+1 .
$$

The right hand side of (3.30) is just $r^{(k+1)}$ from (3.23) and therefore it is $\bar{\gamma}$-closed. One can check that $r^{(k+1)}$ does not depend on undifferentiated ghosts. Indeed, among $h^{(l)}, 0 \leq l \leq k$, only $h^{(0)}$ depends on undifferentiated ghosts but it appears only inside the $*$-commutator. One then finds that $h^{(k+1)}=-\rho r^{(k+1)}$ solves (3.30) because $r^{(k+1)}$ vanishes when $y=z=0$. Furthermore, $h^{(k+1)}$ is also independent on the undifferentiated ghosts so that the construction can be iterated.

In the case of $u(N)$-valued fields it follows from the recursive construction that $h$ also takes values in $u(N)$ because only commutators or anticommutators multiplied by imaginary unit are involved.

Proposition 2. For $h=\sum_{k=0} \vartheta^{k} h^{(k)}$ as in proposition 1 a particular recursive solution $f_{\mu}=\sum_{k=0} \vartheta^{k} f_{\mu}^{(k)}$ to equation (13.18) with $f_{\mu}{ }^{(0)}=A_{\mu}$ is given by

$$
f_{\mu}^{(k+1)}=\rho\left(D_{\mu} h^{(k+1)}+\sum_{i+j+l=k+1}\left[f_{\mu}^{(i)}, h^{(j)}\right]^{(l)}\right), \quad k \geq 0,
$$

with $0 \leq i, j \leq k, 0 \leq l \leq k+1$.

Proof. At zeroth order in $\vartheta$ Eq. (3.18) is satisfied by $f_{\mu}^{0}=f_{\mu}^{(0)}=A_{\mu}$. Assume that we have constructed $f_{\mu}^{k}=\sum_{l=0}^{k} \vartheta^{l} f_{\mu}^{(l)}$ that solves (3.18) to order $k$, i.e.,

$$
\gamma f_{\mu}^{k}-\partial_{\mu} h-\left[f_{\mu}^{k}, h\right]=\vartheta^{k+1} t_{\mu}^{(k+1)}+O(k+2) .
$$

Applying $\gamma+\left[h^{*}, \cdot\right]$ to both sides and using (3.19) one gets at order $k+1$ in $\vartheta$ that $\bar{\gamma} t_{\mu}^{(k+1)}=0$. Explicitly, $t_{\mu}^{(k+1)}$ is given by

$$
t_{\mu}^{(k+1)}=-D_{\mu} h^{(k+1)}-\sum_{i+j+l=k+1}\left[f_{\mu}^{(i)}{ }^{*} h^{(j)}\right]^{(l)}, \quad 0 \leq i, j \leq k, \quad 0 \leq l \leq k+1 .
$$


At order $k+1$ in $\vartheta \mathrm{Eq}$. (3.18) requires:

$$
\begin{gathered}
\bar{\gamma} f_{\mu}^{(k+1)}=D_{\mu} h^{(k+1)}+\sum_{i+j+l=k+1}\left[f_{\mu}^{(i)}{ }^{*} h^{(j)}\right]^{(l)}=-t_{\mu}^{(k+1)} \\
0 \leq i, j \leq k, \quad 0 \leq l \leq k+1 .
\end{gathered}
$$

Thus $f_{\mu}^{(k+1)}=-\rho t_{\mu}^{(k+1)}$ is a particular solution to equation (3.34) because $t_{\mu}^{(k+1)}$ does not depend on undifferentiated ghosts for reasons analogous to those as in the previous proof.

The same arguments as above show that in the case of $u(N)$-valued fields $f_{\mu}$ is also $u(N)$-valued.

\subsubsection{Expansion in homogeneity in the fields}

We now discuss a recursive solution for the SW map based on an expansion according to the homogeneity in the fields. As in the construction of the generating functional the relevant differential is $\gamma^{[0]}$ corresponding to the Abelian theory but now written in terms of unhatted variables. The associated contracting homotopy $\rho^{[0]}$ is defined by (B.27) and satisfies (B.28) with unhatted variables substituting for hatted ones.

Proposition 3. A particular recursive solution $h=\sum_{k=1} h^{[k]}$ to equation (3.19) with $h^{[1]}=C$ is given by

$$
h^{[k]}=-\rho^{[0]}\left(\gamma^{[1]} h^{[k-1]}+\sum_{l=1}^{k-1} \frac{1}{2}\left[h^{[l]}, h^{[k-l]}\right]\right), \quad k \geq 2 .
$$

Proof. At order 1, equation (3.19) is indeed satisfied since $\gamma^{[0]} C=0$. Furthermore, the expression $r^{[2]}=\gamma^{[1]} C+\frac{1}{2}[C, C]$ depends only on differentiated ghosts and hence vanishes when the appropriate variables $y, z$ defined analogously to (B.26) are zero. Suppose that we have constructed $h^{k-1}=\sum_{l=1}^{k-1} h^{[l]}$ satisfying (3.19) up to order $k$, i.e.,

$$
\gamma h^{k-1}+\frac{1}{2}\left[h^{k-1}, h^{k-1}\right]=r^{[k]}+\sum_{m \geq 0} q^{[k+1+m]},
$$

with $r^{[k]}$ and $h^{[k]}, k \geq 2$ in homogeneity $k$ and depending only on differentiated ghosts. Applying $\gamma+\left[h^{k-1} * \cdot \cdot\right]$, the left hand side vanishes identically, which implies for the lowest order on the right hand side that $\gamma^{[0]} r^{[k]}=0$.

In homogeneity degree $k$ equation (3.19) implies:

$$
\gamma^{[0]} h^{k}=-\gamma^{[1]} h^{[k-1]}-\sum_{l=1}^{k-1} \frac{1}{2}\left[h^{[l]}, h^{[k-l]}\right]=r^{[k]} .
$$

Applying $\rho^{[0]}$ to this equation, it follows from (B.28) and the induction hypothesis that $r^{[k]}$ does not depend on undifferentiated ghosts that $h^{[k]}=-\rho^{[0]} r^{[k]}$ is a solution to Eq. (3.37). It remains to be checked that $r^{[k]}$ does not depend on undifferentiated ghosts. Indeed, the 
only possible dependence on undifferentiated ghosts can come from $\gamma^{[1]} h^{[k-1]}+\left[C, h^{[k-1]}\right]$. This dependence cancels between the two terms because: (i) $\gamma^{[1]}=-\left[C, V_{M}\right]^{A} \frac{\partial}{\partial V_{M}^{A}}$ up to terms that involve only differentiated ghosts, with

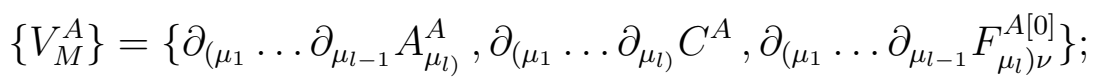

(ii) by construction $h^{[k+1]}$ is a polynomial in matrix-valued fields and their derivatives so that $[C, \cdot]$ satisfies Leibnitz rule.

Explicitly, the quadratic contribution is given by

$$
\begin{aligned}
h^{[2]} & =-\rho^{[0]}\left(\left(\cos \Lambda_{12}-1\right) C^{A} C^{B}\left[T_{A}, T_{B}\right]+i \sin \Lambda_{12} C^{A} C^{B}\left\{T_{A}, T_{B}\right\}\right)= \\
& =-\left(\frac{\vartheta \theta^{\mu \nu}}{2} \frac{\cos \Lambda_{12}-1}{\Lambda_{12}} A_{\mu}^{A} \partial_{\nu} C^{B}\left[T_{A}, T_{B}\right]+i \frac{\vartheta \theta^{\mu \nu}}{2} \frac{\sin \Lambda_{12}}{\Lambda_{12}} A_{\mu}^{A} \partial_{\nu} C^{B}\left\{T_{A}, T_{B}\right\}\right) .
\end{aligned}
$$

Proposition 4. For $h=\sum_{k=1} h^{[k]}$ as in proposition 3, a particular recursive solution $f_{\mu}=\sum_{k=1} f_{\mu}^{[k]}$ of (3.18) with $f_{\mu}^{[1]}=A_{\mu}$ is given by

$$
f_{\mu}^{[k]}=-\rho^{[0]}\left(\gamma^{[1]} f_{\mu}^{[k-1]}-\partial_{\mu} h^{[k]}-\sum_{l=1}^{k-1}\left[f_{\mu}^{[l]}{ }^{*} h^{[k-l]}\right]\right), \quad k \geq 2 .
$$

Proof. At order 1, equation (3.18) is indeed satisfied since $\gamma^{[0]} A_{\mu}=\partial_{\mu} C$. Furthermore, $-\gamma^{[1]} A_{\mu}+\partial_{\mu} h^{[2]}+\left[A_{\mu}{ }^{*} C\right]$ only depends on differentiated ghosts. Suppose that we have constructed $f_{\mu}^{k-1}=\sum_{l=1}^{k-1} f_{\mu}^{[l]}$ such that (3.18) is satisfied up to order $k$,

$$
\gamma f_{\mu}^{k-1}-\partial_{\mu} h-\left[f_{\mu}^{k-1}, h\right]=t_{\mu}^{[k]}+\sum_{m=0} v_{\mu}^{[k+1+m]}
$$

with $t_{\mu}^{[k]}$ depending only on differentiated ghosts. Applying $\gamma+\left[h^{*}, \cdot\right]$ implies to lowest order that $\gamma^{[0]} t_{\mu}^{[k]}=0$. Applying $\rho^{[0]}$, it follows that $t_{\mu}^{[k]}=\gamma^{[0]} \rho^{[0]} t_{\mu}^{[k]}$ which implies that $f_{\mu}^{[k]}=-\rho^{[0]} t_{\mu}^{[k]}$ is a particular solution to equation (3.18) at homogeneity order $k$ :

$$
\gamma^{[0]} f_{\mu}^{[k]}=-\gamma^{[1]} f_{\mu}^{[k-1]}+\partial_{\mu} h^{[k]}-\sum_{l=1}^{k-1}\left[f_{\mu}^{[l]} * h^{[k-l]}\right]=-t_{\mu}^{[k]} .
$$

It remains to be shown that $t_{\mu}^{[k]}$ depends only on differentiated ghosts. Indeed, the only dependence on undifferentiated ghosts can come from the terms $\gamma^{[1]} f_{\mu}^{[k-1]}-\left[f_{\mu}^{[k-1]}, C\right]$ and as before, this dependence cancels between the two terms. Finally, $f_{\mu}^{k}=f_{\mu}^{k-1}+f_{\mu}^{[k]}$ satisfies Eq. (3.18) up to order $k+1$ and the construction can be iterated. 


\subsection{Universal SW map}

The particular SW maps constructed in the previous two sections depend very little on the associative algebra $\mathcal{U}$. Indeed, if as in (3.39), one does not use the multiplication table in $\mathcal{U}$ to simplify the expressions, one can construct a "universal" SW map valid for any $\mathcal{U}$. A SW map for a particular $\mathcal{U}$ is then obtained by using the multiplication table in the final expression. More precisely, this means that one should first construct the SW map for Yang-Mills fields taking values in the free tensor algebra of some sufficiently large vector space. Because each associative algebra $\mathcal{U}$ can be represented as a quotient of such a free tensor algebra modulo some relations, the $\mathrm{SW}$ map for $\mathcal{U}$ can be obtained by using these relations in the SW map for the free tensor algebra.

\subsection{Ambiguities in the SW map}

Some part of the arbitrariness in the SW map was discussed originally in [25, 26, 18. In this section, we are going to derive the most general solution to the SW gauge equivalence condition (3.22). As before indices in parentheses refer to the expansion in the deformation parameter.

Proposition 5. The general solution $f_{\mu}^{\prime}, h^{\prime}$ to equations (3.18)-(3.19) with boundary conditions $f_{\mu}^{\prime(0)}=A_{\mu}, h^{(0)}=C$ is given by the composition

$$
f_{\mu}^{\prime}=f_{\mu}\left[f^{c}[A ; \vartheta] ; \vartheta\right], \quad h^{\prime}=h\left[f^{c}[A ; \vartheta], h^{c}[A, C ; \vartheta] ; \vartheta\right]
$$

where $f_{\mu}, h$ is any particular solution with the same boundary conditions (e.g. the one constructed recursively in the previous section), while $f_{\mu}^{c}, h^{c}$ is the general solution to the "commutative" equations

$$
\begin{gathered}
\gamma h^{c}+\frac{1}{2}\left[h^{c}, h^{c}\right]=0, \\
\gamma f_{\mu}^{c}=\partial_{\mu} h^{c}+\left[f_{\mu}^{c}, h^{c}\right],
\end{gathered}
$$

subject to the boundary condition

$$
f_{\mu}^{c(0)}=A_{\mu}, \quad h^{c(0)}=C .
$$

Proof. Let $F^{\prime}=\int d x\left(\hat{A}_{A}^{\mu \star} f_{\mu}^{\prime A}+\hat{C}_{A}^{*} h^{\prime A}\right)$ and $F=\int d x\left(\hat{A}_{A}^{\mu \star} f_{\mu}^{A}+\hat{C}_{A}^{*} h^{A}\right)$ be the generating functionals for the anticanonical transformation associated with the two SW maps. Denoting by $F^{*}$ the anticanonical transformation corresponding to $F$ (acting on functionals), one has by definition (see eq. (2.4))

$$
F^{*}(\hat{S})=S_{0}^{\mathrm{eff}}+\sum_{k \geq 1} S_{k}^{(0)}
$$

and similarly for $F^{\prime *}$ with $S_{0}^{\text {eff }}$ replaced by some $S_{0}^{\text {eff }}$. It then follows that

$$
F^{\prime *}\left(F^{-1^{*}}\left(\sum_{k \geq 1} S_{k}^{(0)}\right)\right)=\sum_{k \geq 1} S_{k}^{(0)}+\text { antifield-independent terms . }
$$


The antifield dependent part of this equation means that the anticanonical transformation ${F^{\prime *}}^{\prime} \circ F^{-1^{*}}$ preserves the gauge structure of the commutative theory. With

$$
F^{\prime *}\left(F^{-1^{*}}\left(A_{\mu}\right)\right)=f_{\mu}^{c}[A ; \vartheta], \quad F^{\prime *}\left(F^{-1^{*}}(C)\right)=h^{c}[A ; \vartheta](C)
$$

the same argument as in 3.4.1 shows that equations (3.44) and (3.45) hold.

Proposition 6. The general solution to equations (3.44) and (3.45) with boundary conditions (3.46) is given by

$$
\begin{aligned}
& h^{c}=\left.\left(\Lambda^{-1} C \Lambda+\Lambda^{-1} \gamma \Lambda\right)\right|_{C^{A} \rightarrow C^{A}+C^{B} r_{B}^{A}, A_{\mu}^{A} \rightarrow A_{\mu}^{A}+W_{\mu}^{A}+\left(A_{\mu}^{B}+W_{\mu}^{B}\right) r_{B}^{A}}, \\
& f_{\mu}^{c}=\left.\left(\Lambda^{-1} A_{\mu} \Lambda+\Lambda^{-1} \partial_{\mu} \Lambda\right)\right|_{A_{\mu}^{A} \rightarrow A_{\mu}^{A}+W_{\mu}^{A}+\left(A_{\mu}^{B}+W_{\mu}^{B}\right) r_{B}^{A}} .
\end{aligned}
$$

Here $\Lambda=\exp \left(\lambda^{A} T_{A}\right)$ with $\lambda^{A}[A ; \vartheta]$ an arbitrary formal power series in $\vartheta$ with coefficients in local functions; $r$ has the form $r=C^{A} r_{A}^{B}(\vartheta) T_{B}$ and satisfies

$$
\bar{\gamma} r+\frac{1}{2}[r, r]=0
$$

while $W_{\mu}=W_{\mu}^{A}[A ; \vartheta] T_{A}$ satisfies

$$
\bar{\gamma} W_{\mu}=0
$$

The proof is given in appendix C. Notice that equation (3.52) is the Maurer-Cartan equation for $r$ considered as a 1-cochain of the Lie algebra $\mathcal{U}$ with coefficients in the adjoint representation. Notice also that equation (3.53) is equivalent to $W_{\mu}=W_{\mu}(\chi ; \vartheta)$, where the variables $\chi_{\Delta}^{A}$ are defined in appendix $\mathbf{A}$, and the following purely algebraic condition

$$
-\left[C, \chi_{\Delta}^{A}\right] \frac{\partial W_{\mu}}{\partial \chi_{\Delta}^{A}}+\left[C, W_{\mu}\right]=0
$$

Theorem 1. The general solution $f_{\mu}^{\prime}, h^{\prime}$ to equations (3.18) and (3.19) with boundary conditions $f_{\mu}^{\prime(0)}=A_{\mu}, h^{(0)}=C$ is given by

$$
\begin{aligned}
h^{\prime} & =\left.\left(\hat{\Lambda}^{-1} * h * \hat{\Lambda}+\hat{\Lambda}^{-1} * \gamma \hat{\Lambda}\right)\right|_{C^{A} \rightarrow C^{A}+C^{B} r_{B}^{A}, A_{\mu}^{A} \rightarrow A_{\mu}^{A}+W_{\mu}^{A}+\left(A_{\mu}^{B}+W_{\mu}^{B}\right) r_{B}^{A}}, \\
f_{\mu}^{\prime} & =\left.\left(\hat{\Lambda}^{-1} * f_{\mu} * \hat{\Lambda}+\hat{\Lambda}^{-1} * \partial_{\mu} \hat{\Lambda}\right)\right|_{A_{\mu}^{A} \rightarrow A_{\mu}^{A}+W_{\mu}^{A}+\left(A_{\mu}^{B}+W_{\mu}^{B}\right) r_{B}^{A}} .
\end{aligned}
$$

Here, $f_{\mu}, h$ is a particular solution with the same boundary conditions; $\hat{\Lambda}=\exp _{*}\left(\hat{\lambda}^{A} T_{A}\right)$ with $\hat{\lambda}^{A}[A, \vartheta]$ an arbitrary formal power series in $\vartheta$ with coefficients in local functions; $r=C^{A} r_{A}^{B}(\vartheta) T_{B}$ and $W_{\mu}=W_{\mu}^{A}[A ; \vartheta] T_{A}$ satisfy

$$
\bar{\gamma} r+\frac{1}{2}[r, r]=0, \quad \bar{\gamma} W_{\mu}=0 .
$$


The proof is given in appendix $\mathbf{D}$. The general SW map $f_{\mu}^{\prime}$ for the gauge fields can thus be obtained from a particular map $f_{\mu}$, by applying a noncommutative gauge transformation, followed by the substitution $A_{\mu}^{A} \rightarrow A_{\mu}^{A}+A_{\mu}^{B} r_{B}^{A}$ related to a Lie algebra automorphism (see below), and then by the gauge covariant redefinition $A_{\mu}^{A} \rightarrow A_{\mu}^{A}+W_{\mu}^{A}$ of the gauge fields.

The constants $r_{A}^{B}$ which enter here define an automorphism of the Lie algebra (3.21). This is seen by rewriting equation (3.52) in the equivalent form

$$
\gamma \tilde{C}=-\tilde{C} \tilde{C}, \quad \tilde{C}=C+C^{A} r_{A}^{B} T_{B} .
$$

Using that $\gamma C^{A}=-\frac{1}{2} f_{B C}{ }^{A} C^{B} C^{C}$, one readily verifies that equation (3.57) is equivalent to

$$
f_{B C}^{D} e_{D}^{A}=e_{B}^{D} e_{C}^{E} f_{D E}^{A}, \quad e_{A}^{B}=\delta_{A}^{B}+r_{A}^{B} .
$$

This establishes indeed a Lie algebra automorphism $T_{A} \longrightarrow e_{A}^{B} T_{B}$. This automorphism is relevant only if it is an outer automorphism because inner automorphisms can be absorbed by redefinitions of $\Lambda$. In the $u(N)$ case the only outer automorphisms that occur here are rescalings of the $U(1)$ generator (since only automorphisms connected to the identity occur owing to the boundary condition (3.46) $)$.

\subsection{Nontriviality of the deformed action. Chern-Simons theory}

One remaining question is whether the noncommutative $U(N)$ theory as a whole is equivalent to the commutative one, i.e., whether the starting point noncommutative and commutative actions are related through field redefinitions. A necessary condition for triviality is that, at first order, the integrand in (3.15) be on-shell (for the equations of motion of the commutative theory) equal to a total divergence,

$$
L_{0}^{\mathrm{eff}(1)} \approx \partial_{\mu} j_{0}^{\mu}
$$

This condition can be equivalently rewritten as $L_{0}^{\text {eff(1) }} d^{n} x=d j_{0}+\delta k_{1}$ which in turn implies that

$$
L_{0}^{\mathrm{eff}(1)} d^{n} x=d J+s K
$$

(see e.g. [27]). Indeed, it follows from the fact that $\gamma L_{0}^{\mathrm{eff}(1)}=d l_{0}$ that one can find $K$ and $\tilde{J}$ such that $\delta k_{1}=s K+d J$. Namely, using the fact that $\delta f+d g=0$ implies $f=\delta f_{1}+d g_{1}$ for any $f$ of positive pure ghost number and antifield number, one can find $k_{2}$ such that $\delta k_{2}+\gamma k_{1}=d j_{1}$. Proceeding further by induction in antifield number one can construct $K=k_{1}+k_{2}+\ldots$ satisfying $\delta k_{1}=s K+d J$ for some $J$.

In order to prove non-triviality of the noncommutative deformation of $U(N)$ YangMills theory, it is enough to consider the $U(1)$ case. Indeed, by putting to zero all the components of the gauge field except for the one associated to the $u(1)$ factor, triviality of the $U(N)$ theory would imply triviality of the $U(1)$ theory. By applying $s$ to the equation (3.60), we get the descent equation $s J+d m=0$ for the $n-1$ form $J$. This last 
equation then implies, in space-time dimensions strictly greater than 2, (see eq. (13.6) of [27]) that

$$
J=\lambda\left(\star A^{*} C+\star F A\right)+A P(F)+I^{n-1}(\chi)+s(\cdot)+d(\cdot),
$$

where $F=F_{\mu \nu} d x^{\mu} d x^{\nu}, A=A_{\mu} d x^{\mu}$, $\star$ denotes hodge conjugation, and $P(F)$ is a polynomial in the two-form $F$.

It then follows from (3.61) that

$$
L_{0}^{\mathrm{eff}(1)} d^{n} x \approx \lambda(-)^{n} \star F F+F P(F)+d I^{n-1}(\chi) .
$$

Since $L_{0}^{\text {eff(1) }}$ is cubic in $A_{\mu}$ only terms of homogeneity degree 3 can contribute:

$$
L_{0}^{\mathrm{eff}(1)} d^{n} x \approx \alpha F^{3}+d I^{n-1}(\chi) .
$$

In the case where there is no explicit $x$ dependence, this equation implies, by putting to zero the derivatives of the field strength, that

$$
L_{0}^{\mathrm{eff}(1)} d^{n} x=\alpha F^{3} .
$$

Because this is not the case, we conclude that the noncommutative deformation of the action is non trivial, at least in the $x$ independent case and in space-time dimension strictly greater than 2 .

This is to be contrasted with noncommutative $U(N)$ Chern-Simons theory described by the action

$$
\hat{S}_{0}^{C S}=\int \operatorname{Tr}\left(\hat{A} * d \hat{A}+\frac{2}{3} \hat{A} * \hat{A} * \hat{A}\right)
$$

The antifield structure of the solution of the master equation is the same as in the YangMills case. In [30], it has been shown that the SW map transforms the noncommutative $U(N)$ Chern-Simons theory into its commutative counterpart, so that the noncommutative deformation is trivial.

From the point of view of the local BRST cohomology of the commutative theory, this follows directly from the following arguments: for Chern-Simons theory with gauge group $U(N)$, there exists exactly one cohomology class in form degree $n$ and ghost number 0 in the case where $N \geq 2$, and none in the $U(1)$ case (see e.g. [27.) If we denote by $g_{C S}$ the gauge coupling constant that goes with the structure constants of the $s u(N)$ subalgebra, this representative can be chosen to be $\frac{\partial S^{(0)}}{\partial g_{C S}}$. It follows that any consistent deformation of commutative $U(N)$ Chern-Simons theory can be absorbed by a field-antifield redefinition and a redefinition of the gauge coupling. In particular, it follows that there exists a field-antifield redefinition such that

$$
\hat{S}^{C S}\left[\hat{\phi}\left[\phi, \phi^{*} ; \vartheta, g_{C S}\right], \hat{\phi}^{*}\left[\phi, \phi^{*} ; \vartheta, g_{C S}\right] ; \vartheta, \hat{g}_{C S}\right]=S^{(0) C S}\left[\phi, \phi^{*} ; g_{C S}(\vartheta)\right]
$$

with $g_{C S}(\vartheta)=g_{C S}+\vartheta f\left(g_{C S}\right)+\ldots$ In the case where there is no explicit $x$ dependence, dimensional arguments imply that $g_{C S}(\vartheta)=g_{C S}$. 


\section{Local BRST cohomology of noncommutative $U(N)$ Yang-Mills theory}

In this section, we discuss the local BRST cohomology groups of noncommutative $U(N)$ Yang-Mills theory since these groups contain information about anomalies, counterterms, observables, symmetries and conservation laws of the theory (for details see [27] and references therein). By using a SW map, these groups can be most conveniently analyzed in terms of the effective Yang-Mills theory because only the part of the BRST cohomology groups that pertains to the dynamics differs from that of standard Yang-Mills theory. The BRST cohomology groups for effective Yang-Mills theories have been discussed in [27.

\subsection{Basic considerations}

Let $\Omega$ be the space of local forms and $\Omega[[\vartheta]]=\Omega \otimes[[\vartheta]]$ be the space of formal power series in the deformation parameter with values in $\Omega$. We want to analyze $H^{k, p}(\hat{s} \mid d, \Omega[[\vartheta]])$, the local BRST cohomology group in ghost number $k$ and form degree $p$. An element of $H^{k, p}(\hat{s} \mid d, \Omega[[\vartheta]])$ is an equivalence class of a local $p$-form $a^{k, p}$ of ghost number $k$ satisfying the cocycle condition

$$
\hat{s} a^{p, k}+d a^{p+1, k-1}=0
$$

modulo the equivalence relation

$$
a^{k, p} \sim a^{k, p}+\hat{s} b^{k-1, p}+d b^{k, p-1} .
$$

Standard arguments using an expansion in the deformation parameter allow one to show that $H^{k, p}(\hat{s} \mid d, \Omega[[\vartheta]]) \subset H^{k, p}\left(\hat{s}^{(0)} \mid d, \Omega\right) \otimes[[\vartheta]]$. In order to describe $H^{k, p}(\hat{s} \mid d)$ it is thus sufficient to check which elements from $H^{k, p}\left(\hat{s}^{(0)} \mid d, \Omega\right)$ can be completed to elements in $H^{k, p}(\hat{s} \mid d, \Omega[[\vartheta]])$. The same considerations apply to the groups $H(\hat{\gamma} \mid d, \Omega[[\vartheta]])$ and $H(\hat{\delta} \mid d, \Omega[[\vartheta]])$.

Let $\hat{z}^{A}$ denote collectively all the fields, ghosts, and antifields of the theory. Consider the invertible change of variables $\hat{z}^{A}=\hat{z}^{A}[z ; \vartheta]$, with $\hat{z}^{A}[z ; \vartheta]$ formal power series in $\vartheta$ with values in local functions depending on the new variables $z^{B}$. This change of variables is extended to the derivatives $\hat{z}_{, \mu_{1} \ldots \mu_{k}}^{A}$ in such a way that the expression of the total derivative $\partial_{\mu}=\frac{\partial}{\partial x^{\mu}}+\hat{z}_{, \mu}^{A} \frac{\partial}{\partial \hat{z}^{A}}+\ldots$ is the same in the new variables $z^{B}$ as it was in the old variables. In terms of the new variables, the expression for the differential $\hat{s}$ becomes

$$
\hat{s} z^{B}=\left.\sum_{k=0}\left[\left(\partial_{\mu_{1}} \ldots \partial_{\mu_{k}} \hat{s} \hat{z}^{A}\right)\left(\frac{\partial z^{B}}{\partial \hat{z}_{, \mu_{1} \ldots \mu_{k}}^{A}}\right)\right]\right|_{\hat{z}=\hat{z}[z]}
$$

\subsection{Local BRST cohomology in the effective theory}

Let us now apply the considerations of the previous subsection to the case of noncommutative $U(N)$ Yang-Mills theory and use as a change of variables a particular SW map. 
We denote the BRST differential in terms of the new unhatted variables by $s^{\text {eff }}$. By definition of a SW map, $s^{\text {eff }}=\delta^{\text {eff }}+\gamma$. The differentials $\gamma$ and $\delta^{\text {eff }}$ coincide with $\hat{\gamma}^{(0)}$ respectively $\hat{\delta}^{(0)}$ in terms of unhatted fields and antifields, except for the action of $\delta^{\text {eff }}$ on the antifields $A_{A}^{* \mu}$, which contains correction terms due to the higher dimensional gauge invariant interactions,

$$
\delta^{\mathrm{eff}} A_{A}^{* \mu}=\frac{\delta S_{0}^{\mathrm{eff}}[A]}{\delta A_{\mu}^{A}}=\frac{\delta S_{0}^{(0)}[A]}{\delta A_{\mu}^{A}}+O(\vartheta) .
$$

As a consequence, local BRST cohomology groups like $H(\hat{\gamma} \mid d, \Omega[[g]])$ that do not involve the dynamics, are completely determined by the local BRST cohomology groups of standard $U(N)$ Yang-Mills theory,

$$
H(\hat{\gamma} \mid d, \Omega[[g]]) \simeq H(\gamma \mid d, \Omega) \otimes[[\vartheta]] .
$$

In particular, in ghost number 1 and form degree $n$, this means that the potential chiral anomalies of noncommutative $U(N)$ Yang-Mills theory (treated as an effective theory) are directly related to the commutative ones.

How the dynamics of effective Yang-Mills theories enters the local BRST cohomology groups has been analyzed in some detail in [27. In particular, this analysis also applies to the effective field theory formulation of noncommutative $U(N)$ Yang-Mills theories.

In the following subsection, we will only briefly discuss some BRST cohomology classes of the standard $U(N)$ Yang-Mills theory that involve the dynamics and get obstructed under the noncommutative deformation.

\subsection{Breaking of Poincaré invariance in noncommutative defor- mation}

Local BRST cohomology classes that involve the dynamics are for instance those in ghost number -1 and form degree $n$. They contain the information about the global symmetries and the associated conserved currents. In particular, we concentrate on the Poincaré invariance, or, in 4 space-time dimensions, the conformal invariance of standard Yang-Mills theory.

Standard homological arguments (see e.g. [27]) show that

$$
H^{-1, n}(\hat{s} \mid d, \Omega[[\vartheta]]) \simeq H_{1}^{n}(\hat{\delta} \mid d, \Omega[[\vartheta]]) .
$$

In turn, by the same reasoning that relates the $\hat{s}$ to the $s^{\text {eff }}$ cohomology, this group is given by $H_{1}^{n}(\hat{\delta} \mid d, \Omega[[\vartheta]]) \simeq H_{1}^{n}\left(\delta^{\text {eff }} \mid d, \Omega[[\vartheta]]\right)$. For representatives $\omega_{1}^{n}=Q_{\mu}^{A} A_{A}^{* \mu} d^{n} x$, the cocycle condition of this last group determines the global symmetries and the associated Noether currents:

$$
\delta^{\mathrm{eff}}\left(A_{A}^{* \mu} Q_{\mu}^{A} d^{n} x\right)+d j=0 \Longleftrightarrow \frac{\delta L^{\mathrm{eff}}}{\delta A_{\mu}^{A}} Q_{\mu}^{A}+\partial_{\mu} j^{\mu}=0 .
$$

By taking the coboundary condition into account, $H_{1}^{n}\left(\delta^{\mathrm{eff}} \mid d, \Omega[[\vartheta]]\right)$ can be shown to correspond to the non trivial global symmetries (respectively the non trivial conserved currents) associated to $L_{0}^{\text {eff }}$. 
In space-time dimensions $n$ strictly greater than 2 , the general solution to the conformal Killing equation

$$
\eta_{\mu \rho} \partial_{\nu} \xi^{\rho}+\eta_{\nu \rho} \partial_{\mu} \xi^{\rho}=\frac{2}{n} \eta_{\mu \nu} \partial_{\rho} \xi^{\rho}
$$

reads as

$$
\xi^{\mu}=a^{\mu}+\lambda_{\nu}^{\mu} x^{\nu}+c x^{\mu}+b^{\nu} \eta_{\nu \rho} x^{\rho} x^{\mu}-\frac{1}{2} b^{\mu} \eta_{\nu \rho} x^{\nu} x^{\rho} .
$$

Here, the constants $a^{\mu}$ correspond to translations, $\lambda_{\nu}^{\mu}$ with $\lambda_{\mu \nu} \equiv \eta_{\mu \rho} \lambda_{\nu}^{\rho}=-\lambda_{\nu \mu}$ to Lorentz transformations, $c$ to dilatations and $b^{\mu}$ to special conformal transformations. The conformal transformations act on the potentials and the associated curvatures as Lie derivatives,

$$
\begin{aligned}
\delta_{\xi} A_{\mu} & =\mathcal{L}_{\xi} A_{\mu}=\xi^{\rho} \partial_{\rho} A_{\mu}+A_{\rho} \partial_{\mu} \xi^{\rho} \\
\delta_{\xi} F_{\mu \nu} & =\mathcal{L}_{\xi} F_{\mu \nu}=\xi^{\rho} \partial_{\rho} F_{\mu \nu}+F_{\rho \nu} \partial_{\mu} \xi^{\rho}+F_{\mu \rho} \partial_{\nu} \xi^{\rho}
\end{aligned}
$$

Because the Poincaré, respectively the conformal transformations in 4 dimensions, are symmetries of commutative Yang-Mills theory, taking $Q_{\mu}^{(0) A}=\delta_{\xi} A_{\mu}^{A}$ with the appropriate $\xi$ allows one to satisfy equation (4.7) to order zero in $\vartheta$ for some $j^{(0) \mu}$.

In order for these symmetries to survive the noncommutative deformation to first order in $\vartheta$, one needs to find $Q_{\mu}^{(1) A}, j^{(1) \mu}$ such that

$$
\frac{\delta L_{0}^{\mathrm{eff}(1)}}{\delta A_{\mu}^{A}} \delta_{\xi} A_{\mu}+\frac{\delta L_{0}^{\mathrm{eff}(0)}}{\delta A_{\mu}^{A}} Q_{\mu}^{(1) A}+\partial_{\mu} j^{(1) \mu}=0 .
$$

Explicitly, the first term reduces to

$$
\begin{aligned}
\frac{\delta L_{0}^{\mathrm{eff}(1)}}{\delta A_{\mu}^{A}} \delta_{\xi} A_{\mu}=\frac{i \eta^{\mu \rho} \eta^{\nu \sigma}}{2 \kappa^{2}}\left(\theta^{\alpha \sigma} \partial_{\sigma} \xi^{\beta}+\right. & \left.\theta^{\sigma \beta} \partial_{\sigma} \xi^{\alpha}\right) \\
& \operatorname{Tr}\left(-\frac{1}{2} F_{\mu \nu}\left\{F_{\alpha \rho}, F_{\beta \sigma}\right\}+\frac{1}{8} F_{\alpha \beta}\left\{F_{\mu \nu}, F_{\rho \sigma}\right\}\right)
\end{aligned}
$$

where we used that $\xi$ is a conformal Killing vector. This expression coincides with $L_{0}^{\text {eff(1) }}$ with $\theta^{\alpha \beta}$ replaced by $\theta^{\alpha \sigma} \partial_{\sigma} \xi^{\beta}+\theta^{\sigma \beta} \partial_{\sigma} \xi^{\alpha}$. In its turn, $L_{0}^{\text {eff(1) }}$ was proved in subsection 3.7 to be non trivial, i.e., not proportional to equations of motion modulo a total derivative ${ }^{4}$. Thus we conclude that Eq. (4.11) admits solutions $Q_{\mu}^{(1) A}, j^{(1) \mu}$ iff

$$
\theta^{\alpha \sigma} \partial_{\sigma} \xi^{\beta}+\theta^{\sigma \beta} \partial_{\sigma} \xi^{\alpha}=0
$$

i.e., if $\xi$ preserves $\theta$.

Let us assume that $\theta$ is nondegenerate (symplectic). It then follows that $\partial_{\mu} \xi^{\mu}=0$. This means in particular in 4 dimensions that the dilatations and the special conformal symmetries are obstructed. Thus we can assume that

$$
\xi^{\mu}=a^{\mu}+\lambda_{\nu}^{\mu} x^{\nu}
$$

\footnotetext{
${ }^{4}$ For the special conformal transformations, the arguments given in subsection 3.7 have to be extended to local forms depending explicitly on $x$ since $\partial_{\mu} \xi^{\nu}$ depends linearly on $x$.
} 
The Killing condition together with (4.13) then require

$$
\begin{aligned}
\eta^{\mu \rho} \lambda_{\rho}^{\nu}+\eta^{\rho \nu} \lambda_{\rho}^{\mu} & =0, \\
\theta^{\mu \rho} \lambda_{\rho}^{\nu}+\theta^{\rho \nu} \lambda_{\rho}^{\mu} & =0 .
\end{aligned}
$$

Conversely, any $\xi$ of the form $\xi^{\mu}=a^{\mu}+\lambda_{\nu}^{\mu} x^{\nu}$ satisfying the previous equations defines a symmetry of $L_{0}^{\text {eff }}$. Indeed, since $\xi$ is at most linear in $x$, we have $\left[L_{\xi}, \partial_{\mu}\right]=0$. For any function $f(\eta, \theta,[A])$ with all space-time indices of $A_{\mu}^{A}$ and their derivatives contracted by either $\theta^{\mu \nu}$ or $\eta^{\mu \nu}$, we then get

$$
\delta_{\xi} f=\sum_{k=0} \partial_{\rho_{1}} \ldots \partial_{\rho_{k}}\left(\mathcal{L}_{\xi} A_{\mu}^{A}\right) \frac{\partial f}{\partial A_{\mu, \rho_{1} \ldots \rho_{k}}^{A}}=\mathcal{L}_{\xi} f=\partial_{\rho}\left(\xi^{\rho} f\right),
$$

where the second equality holds because $\mathcal{L}_{\xi} \theta=0=\mathcal{L}_{\xi} \eta$ on account of (4.15) and the last equality follows from $\partial_{\rho} \xi^{\rho}=0$. Thus $L_{0}^{\text {eff }}$ is invariant up to a total derivative to all orders in $\vartheta$, which means that (4.7) can be satisfied with $Q_{\mu}^{A}=Q_{\mu}^{(0) A}=\delta_{\xi} A_{\mu}^{A}$. Hence, only the Poincaré transformations that satisfy in addition $\mathcal{L}_{\xi} \theta=0$ are unobstructed and define global symmetries of $L_{0}^{\mathrm{eff}}$.

In fact, the argument leading to (4.16) can be used to show directly without using the SW map that, in the case where $\theta$ is non degenerate, the Poincaré transformations satisfying $\mathcal{L}_{\xi} \theta=0$ define global symmetries of $\hat{L}_{0}$.

Let us now discuss in more detail the Lie subalgebra of Lorentz transformations that satisfy $\mathcal{L}_{\xi} \theta=0$ for a non degenerate $\theta$. This Lie subalgebra crucially depends on the respective position of the matrices $\eta^{\mu \nu}$ and $\theta^{\mu \nu}$. It is useful to introduce the linear operators

$$
J_{\mu}^{\nu}=\theta_{\mu \rho} \eta^{\rho \nu}, \quad K_{\mu}^{\nu}=J_{\mu}^{\rho} J_{\rho}^{\nu},
$$

with $\theta_{\mu \rho} \theta^{\rho \nu}=\delta_{\mu}^{\nu}$ and $K_{\mu \nu} \equiv \eta_{\mu \rho} K_{\nu}^{\rho}=K_{\nu \mu}$.

Proposition 7. If the operator $K$ can be diagonalized over $\mathbb{R}$, with $t_{1}, \ldots, t_{N}$ denoting its distinct eigenvalues, then the eigenspaces of $K$ with eigenvalues $t_{\alpha}$ are even-dimensional of dimension $2 n_{\alpha}$ and the subalgebra $\mathfrak{g}$ of Lorentz transformations satisfying $\mathcal{L}_{\xi} \theta=0$ decomposes as

$$
\mathfrak{g} \simeq \oplus_{\alpha=1}^{N} \mathfrak{g}_{\alpha}
$$

where for negative $t_{\alpha}, \mathfrak{g}_{\alpha}$ is $u\left(n_{\alpha}^{+}, n_{\alpha}^{-}\right)$with $n_{\alpha}^{+}+n_{\alpha}^{-}=n_{\alpha}$, while for positive $t_{\alpha}, \mathfrak{g}_{\alpha}$ is $\operatorname{gl}\left(n_{\alpha}, \mathbb{R}\right)$.

The proof of the proposition is given in the appendix $\mathbf{E}$. In particular, if $\eta$ is Euclidean, $K$ can be diagonalized and

$$
\mathfrak{g} \simeq \oplus_{\alpha=1}^{N} u\left(n_{\alpha}\right), \quad \sum_{\alpha=1}^{N} n_{\alpha}=\frac{n}{2},
$$

where $n$ denotes the dimension of the Euclidean space (which is even because $\theta$ is non degenerate). If all the eigenvalues of $K$ coincide, the symmetry algebra is $u(n / 2)$ of maximal dimension $n^{2} / 4$. 
In the 4-dimensional Minkowski case with canonical $\theta$,

$$
\eta=\left(\begin{array}{cccc}
-1 & 0 & 0 & 0 \\
0 & 1 & 0 & 0 \\
0 & 0 & 1 & 0 \\
0 & 0 & 0 & 1
\end{array}\right), \quad \theta=\left(\begin{array}{cccc}
0 & 0 & 1 & 0 \\
0 & 0 & 0 & 1 \\
-1 & 0 & 0 & 0 \\
0 & -1 & 0 & 0
\end{array}\right)
$$

$K$ is diagonalizable and $\mathfrak{g} \simeq g l(1, \mathbb{R}) \oplus u(1)$ is a 2-dimensional Abelian algebra.

To complete the discussion let us briefly consider the case where $\theta$ is degenerate. This case is more difficult because there is no longer a suitable tensor $J$ as in (4.17). For simplicity, let us discuss the case where the matrices $\eta^{\mu \nu}$ and $\theta^{\mu \nu}$ take the following form:

$$
\eta^{\mu \nu}=\left(\begin{array}{cc}
\eta^{i j} & 0 \\
0 & \eta^{a b}
\end{array}\right), \quad \theta^{\mu \nu}=\left(\begin{array}{cc}
0 & 0 \\
0 & \theta^{a b}
\end{array}\right) .
$$

This covers in particular Minkowski space-time where the time coordinate is "commuting", i.e., for $\eta=\operatorname{diag}(-1,1, \ldots, 1)$ and $\theta^{0 \mu}=0$. The condition (4.13) takes the form

$$
\theta^{a c} \partial_{c} \xi^{b}+\theta^{c b} \partial_{c} \xi^{a}=0, \quad \partial_{c} \xi^{i}=0 .
$$

The first equation can be solved by $\xi^{a}=\theta^{a c} \partial_{c} H$ for some function $H$. At the same time conformal Killing condition (4.8) implies that

$$
\left(\partial_{i} \xi^{i}+\partial_{c} \xi^{c}\right) \eta^{a b}=\frac{2}{n}\left(\eta^{a c} \partial_{c} \xi^{b}+\eta^{c b} \partial_{c} \xi^{a}\right), \quad \partial_{i} \xi^{c}=0 .
$$

Multiplying the first equation by $\eta_{a b}$ and substituting $\xi^{a}=\theta^{a c} \partial_{c} H$ one gets

$$
\partial_{\mu} \xi^{\mu}=\partial_{i} \xi^{i}+\partial_{c} \xi^{c}=0,
$$

which again implies that dilatations and special conformal symmetries are obstructed in 4 dimensions. The problem can now be reduced to the non-degenerate case already solved in proposition 7 the algebra $\mathfrak{g}$ is the direct sum $\mathfrak{g}=\mathfrak{g}_{1} \oplus \mathfrak{g}_{2}$, where $\mathfrak{g}_{1}$ are the Lorentz transformations associated to the metric $\eta^{i j}$ and $\mathfrak{g}_{2}$ is the subalgebra of Lorentz transformations preserving both $\eta^{a b}$ and the non-degenerate $\theta^{a b}$.

\section{Acknowledgments}

The work of GB and MG is supported in part by the "Actions de Recherche Concertées" of the "Direction de la Recherche Scientifique-Communauté Française de Belgique", by a "Pôle d'Attraction Interuniversitaire" (Belgium), by IISN-Belgium, convention 4.4505.86, by the INTAS grant 00-00262, and by the European Commission RTN program HPRN-CT00131, in which the authors are associated to K. U. Leuven. GB is also supported by Proyectos FONDECYT 1970151 and 7960001 (Chile), while MG is supported by RFBR grants 01-01-00906 and 02-01-06096. 


\section{Appendices}

\section{Appendix A: Proof of (3.7)}

Using explicitly the decomposition according to the antifield number, defined by assigning degree 1 and 2 to $A_{A}^{* \mu}$ and $C_{A}^{*}$ respectively, with $A_{\mu}^{A}$ and $C^{A}$ carrying zero degree, the commutative BRST differential $s$ decomposes as

$$
s=\delta+\gamma
$$

where $\delta$ and $\gamma$ are of antifield numbers -1 and 0, respectively. Explicitly,

$$
\begin{aligned}
\gamma A_{\mu} & =D_{\mu} C, & \gamma C & =-C C, \\
\gamma A_{A}^{* \mu} & =f_{B A}{ }^{C} C^{B} A_{C}^{* \mu}, & \gamma C_{A}^{*} & =f_{B A}{ }^{C} C^{B} C_{C}^{*}, \\
\delta A_{\mu} & =0, & \delta C & =0, \\
\delta A_{A}^{* \mu} & =\frac{1}{\kappa^{2}} \operatorname{Tr}\left(T_{A} D_{\nu} F^{\nu \mu}\right), & \delta C_{A}^{*} & =-\partial_{\mu} A_{A}^{* \mu}+f_{B A}{ }^{C} A_{\mu}^{B} A_{C}^{* \mu},
\end{aligned}
$$

where $f_{A B}{ }^{C}$ are the structure constants defined by $\left[T_{A}, T_{B}\right]=f_{A B}{ }^{C} T_{C}$.

An element $\tilde{S}^{(k+1)} \in \mathfrak{S}$ contains parts with antifield numbers 0,1 and 2 :

$$
\tilde{S}^{(k+1)}=\tilde{S}_{0}^{(k+1)}+\tilde{S}_{1}^{(k+1)}+\tilde{S}_{2}^{(k+1)} .
$$

Eq. (3.6) decomposes thus into terms with antifield numbers 2, 1 and 0 :

$$
\gamma \tilde{S}_{2}^{(k+1)}=0, \gamma \tilde{S}_{1}^{(k+1)}+\delta \tilde{S}_{2}^{(k+1)}=0, \gamma \tilde{S}_{0}^{(k+1)}+\delta \tilde{S}_{1}^{(k+1)}=0 .
$$

Along the lines of [24, 32], we introduce the following variables $y^{\alpha}, z^{\alpha}, w^{i}$ as new coordinates in the space of fields, antifields and their derivatives:

$$
\begin{aligned}
& \left\{y^{\alpha}\right\}=\left\{\partial_{\left(\nu_{1}\right.} \ldots \partial_{\nu_{l}} A_{\mu)}^{A}\right\}, \quad\left\{z^{\alpha}\right\}=\left\{\partial_{\left(\nu_{1}\right.} \ldots \partial_{\nu_{l}} D_{\mu)} C^{A}\right\} \\
& \left\{w^{i}\right\}=\left\{C^{A}, D_{\left(\nu_{1}\right.} \ldots D_{\nu_{l}} F_{\mu) \lambda}^{A}, D_{\left(\nu_{1}\right.} \ldots D_{\left.\nu_{l}\right)} A_{A}^{* \mu}, D_{\left(\nu_{1}\right.} \ldots D_{\left.\nu_{l}\right)} C_{A}^{*}\right\}
\end{aligned}
$$

where $l=0,1, \ldots$ These variables are independent and complete in the sense that every local function of the fields, antifields and their derivatives can be uniquely expressed in terms of them. ${ }^{5}$ We define a homotopy operator $\rho$ on functions of these variables by

$$
\rho f(y, z, w)=\int_{0}^{1} \frac{d t}{t} y^{\alpha} \frac{\partial f(t y, t z, w)}{\partial z^{\alpha}} .
$$

It satisfies

$$
\{\gamma, \rho\} f(y, z, w)=f(y, z, w)-f(0,0, w)
$$

When $f(y, z, w)$ is $\gamma$-closed this relation yields in particular:

$$
\gamma f(y, z, w)=0 \quad \Rightarrow \quad f(y, z, w)=f(0,0, w)+\gamma \rho f(y, z, w) .
$$

\footnotetext{
${ }^{5}$ It is the independence of these variables which avoids constraints as encountered in 17 . 18.
} 
It states that the cohomology of $\gamma$ can be constructed solely in terms of the $w$ 's - the dependence of $\gamma$-cocycles on the $y$ 's and $z$ 's is trivial. We stress that this holds for local functions - in general it does not hold for local functionals whose integrands are $\gamma$-closed only up to total derivatives, the reason being that $\rho$ does not commute with $\partial_{\mu}$. Nevertheless we can use (A.11) to analyze Eqs. (A.7) thanks to the fact that $\tilde{S}_{2}^{(k+1)}=$ $\int d^{n} x \omega_{2}^{(k+1)}$ and $\tilde{S}_{1}^{(k+1)}$ are linear in antifields. Indeed, consider the first equation of (A.7). Since the integrand $\omega_{2}^{(k+1)}$ is linear in the undifferentiated antifields $C_{A}^{*}$, so is $\gamma \omega_{2}^{(k+1)}$ (owing to $\gamma C_{A}^{*}=f_{B A}{ }^{C} C^{B} C_{C}^{*}$ ). Hence $\gamma \omega_{2}^{(k+1)}$ cannot be a (nonvanishing) total derivative and $\gamma \widetilde{S}_{2}^{(k+1)}=0$ implies thus that it vanishes, $\gamma \omega_{2}^{(k+1)}=0$. Because $\omega_{2}^{(k+1)}$ depends on the ghosts only via their derivatives, it vanishes at $y^{\alpha}=z^{\alpha}=0$ when expressed in terms of the variables $y^{\alpha}, z^{\alpha}, w^{i}$ because $\partial_{\alpha} C=D_{\alpha} C-\left[A_{\alpha}, C\right]$ itself vanishes at $y^{\alpha}=z^{\alpha}=0$. Using (A.11) we conclude $\omega_{2}^{(k+1)}=\gamma \rho \omega_{2}^{(k+1)}$, which yields

$$
\tilde{S}_{2}^{(k+1)}=\gamma \tilde{\Xi}_{2}^{(k+1)}, \quad \tilde{\Xi}_{2}^{(k+1)}=\int d^{n} x \rho \omega_{2}^{(k+1)} \in \mathfrak{S} .
$$

Using this in the second equation (A.7), the latter yields

$$
\gamma\left(\tilde{S}_{1}^{(k+1)}-\delta \tilde{\Xi}_{2}^{(k+1)}\right)=0
$$

owing to $\{\delta, \gamma\}=0$. The functional $\tilde{S}_{1}^{(k+1)}-\delta \tilde{\Xi}_{2}^{(k+1)}=\int d^{n} x \tilde{\eta}_{1}^{(k+1)}$ depends linearly on the antifields $A_{A}^{* \mu}$ and their derivatives (derivatives of $A_{A}^{* \mu}$ occur because $\delta \Xi_{2}^{(1)}$ contains $\left.\delta C_{A}^{*}=-\partial_{\mu} A_{A}^{* \mu}+\ldots\right)$. Using integration by parts that remove all derivatives of $A_{A}^{* \mu}$, this functional can be seen to belong to $\mathfrak{S}$ and takes the form $\int d^{n} x \eta_{1}^{(k+1)}$ with $\eta_{1}^{(k+1)}$ not involving any derivatives of $A_{A}^{* \mu}$.

As before, from $\gamma \int d^{n} x \eta_{1}^{(k+1)}=0$ we conclude that $\gamma \eta_{1}^{(k+1)}=0$ because $\eta_{1}^{(k+1)}=0$ is linear in antifields and does not contain any derivatives of them. One then deduces that $\eta_{1}^{(k+1)}$ vanishes at $y^{\alpha}=z^{\alpha}=0$ when expressed in terms of the variables $y^{\alpha}, z^{\alpha}, w^{i}$ because it depends on the ghosts only via their derivatives. Hence we can use (A.11) again, and conclude that $\eta_{1}^{(k+1)}=\gamma \rho \eta_{1}^{(k+1)}$ which yields

$$
\tilde{S}_{1}^{(k+1)}-\delta \tilde{\Xi}_{2}^{(k+1)}=\gamma \tilde{\Xi}_{1}^{(k+1)}, \quad \tilde{\Xi}_{1}^{(k+1)}=\int d^{n} x \rho \eta_{1}^{(k+1)} \in \mathfrak{S} .
$$

Using eqs. (A.12) and (A.14) in (A.6), we obtain

$$
\tilde{S}^{(k+1)}=S_{0}^{\mathrm{eff}(k+1)}[A]+s \tilde{\Xi}^{(k+1)},
$$

with

$$
\begin{aligned}
& \tilde{\Xi}^{(k+1)}=\tilde{\Xi}_{1}^{(k+1)}+\tilde{\Xi}_{2}^{(k+1)} \in \mathfrak{S}, \\
& S_{0}^{\operatorname{eff}(k+1)}[A]=\quad \tilde{S}_{0}^{(k+1)}-\delta \tilde{\Xi}_{1}^{(k+1)} .
\end{aligned}
$$




\section{Appendix B: Explicit construction of $\hat{\Xi}$ and $\hat{B}_{0}$}

The decomposition of $\partial \hat{S} / \partial \vartheta$ according to the antifield number reads:

$$
\begin{aligned}
\frac{\partial \hat{S}}{\partial \vartheta} & =\frac{\partial \hat{S}_{0}}{\partial \vartheta}+\frac{\partial \hat{S}_{1}}{\partial \vartheta}+\frac{\partial \hat{S}_{2}}{\partial \vartheta} \\
\frac{\partial \hat{S}_{0}}{\partial \vartheta} & =\frac{-i \theta^{\alpha \beta}}{2 \kappa^{2}} \int d^{n} x \operatorname{Tr}\left(\hat{F}^{\mu \nu} \partial_{\alpha} \hat{A}_{\mu} * \partial_{\beta} \hat{A}_{\nu}\right) \\
\frac{\partial \hat{S}_{1}}{\partial \vartheta} & =\frac{i \theta^{\alpha \beta}}{2} \int d^{n} x \hat{A}_{A}^{* \mu}\left\{\partial_{\alpha} \hat{A}_{\mu}^{*}, \partial_{\beta} \hat{C}\right\}^{A} \\
\frac{\partial \hat{S}_{2}}{\partial \vartheta} & =\frac{i \theta^{\alpha \beta}}{2} \int d^{n} x \hat{C}_{A}^{*}\left(\partial_{\alpha} \hat{C} * \partial_{\beta} \hat{C}\right)^{A}
\end{aligned}
$$

while $\hat{s} \frac{\partial \hat{S}}{\partial \vartheta}=0$ decomposes according to:

$$
\hat{\gamma} \frac{\partial \hat{S}_{2}}{\partial \vartheta}=0, \quad \hat{\gamma} \frac{\partial \hat{S}_{1}}{\partial \vartheta}+\hat{\delta} \frac{\partial \hat{S}_{2}}{\partial \vartheta}=0, \quad \hat{\gamma} \frac{\partial \hat{S}_{0}}{\partial \vartheta}+\hat{\delta} \frac{\partial \hat{S}_{1}}{\partial \vartheta}=0
$$

In order to analyze these equations, we use a decomposition according to the homogeneity in all (hatted) fields and antifields.

We start from the first equation of (B.5). To remove the integral, we take the variational derivative with respect to $\hat{C}_{A}^{*}$. This yields

$$
0=\frac{\delta}{\delta \hat{C}_{A}^{*}}\left[\hat{\gamma} \frac{\partial \hat{S}_{2}}{\partial \vartheta}\right]=\left(\hat{\gamma} \eta+\left[\hat{C}^{*}, \eta\right]\right)^{A}
$$

where

$$
\eta=\frac{i \theta^{\alpha \beta}}{2} \partial_{\alpha} \hat{C} * \partial_{\beta} \hat{C}
$$

At lowest order in the fields, Eq. (B.6) yields:

$$
\hat{\gamma}^{[0]} \eta=0 \text {. }
$$

For $\eta$ of the form (B.7), we will show below that $\hat{\gamma}^{[0]}$ can be "inverted", i.e. that there exists $\xi$ such that

$$
\eta=\hat{\gamma}^{[0]} \xi
$$

This equation defines $\xi$ only up to a $\hat{\gamma}^{[0]}$-cocycle. A convenient choice, to be discussed in more detail below, turns out to be

$$
\xi=\frac{i \theta^{\alpha \beta}}{4}\left\{\hat{A}_{\alpha}^{*}, \partial_{\beta} \hat{C}\right\}
$$

That this $\xi$ actually satisfies $(\underline{B .9})$ can be directly checked. We thus obtain

$$
\partial \hat{S}_{2} / \partial \vartheta=\int d^{n} x \hat{C}_{A}^{*} \eta^{A}=\hat{\gamma}^{[0]} \int d^{n} x \hat{C}_{A}^{*} \xi^{A},
$$


where we can replace $\hat{\gamma}^{[0]}$ with $\hat{\gamma}$ because of $\hat{\gamma}^{[1]} \int d^{n} x \hat{C}_{A}^{*} \xi^{A}=0$ (the latter holds because $\left.\hat{\gamma}^{[1]} \xi=-\left[\hat{C}^{*}, \xi\right]\right)$ :

$$
\frac{\partial \hat{S}_{2}}{\partial \vartheta}=\hat{\gamma} \hat{\Xi}_{2}, \quad \hat{\Xi}_{2}=\frac{i \theta^{\alpha \beta}}{4} \int d^{n} x \hat{C}_{A}^{*}\left\{\hat{A}_{\alpha}^{*}, \partial_{\beta} \hat{C}\right\}^{A}
$$

Using (B.12) in the second equation (B.5), we obtain:

$$
\hat{\gamma}\left[\frac{\partial \hat{S}_{1}}{\partial \vartheta}-\hat{\delta} \hat{\Xi}_{2}\right]=0
$$

We now proceed as before and apply the variational derivative with respect to $\hat{A}_{A}^{* \mu}$. This yields:

$$
0=\frac{\delta}{\delta \hat{A}_{A}^{* \mu}}\left(\hat{\gamma}\left[\frac{\partial \hat{S}_{1}}{\partial \vartheta}-\hat{\delta} \hat{\Xi}_{2}\right]\right)=-\left(\hat{\gamma} \eta_{\mu}+\left[\hat{C}^{*}, \eta_{\mu}\right]\right)^{A}
$$

where

$$
\eta_{\mu}=\frac{i \theta^{\alpha \beta}}{4}\left(2\left\{\partial_{\alpha} \hat{A}_{\mu}^{*}, \partial_{\beta} \hat{C}\right\}-\partial_{\mu}\left\{\hat{A}_{\alpha}^{*}, \partial_{\beta} \hat{C}\right\}-\left[\hat{A}_{\mu}, *\left\{\hat{A}_{\alpha}^{*}, \partial_{\beta} \hat{C}\right\}\right]\right)
$$

The decomposition of $\eta_{\mu}$ reads thus

$$
\begin{aligned}
& \eta_{\mu}=\eta_{\mu}^{[2]}+\eta_{\mu}^{[3]}, \\
& \eta_{\mu}^{[2]}=\frac{i \theta^{\alpha \beta}}{4}\left(2\left\{\partial_{\alpha} \hat{A}_{\mu}{ }^{*}, \partial_{\beta} \hat{C}\right\}-\partial_{\mu}\left\{\hat{A}_{\alpha}, \partial_{\beta} \hat{C}\right\}\right), \\
& \eta_{\mu}^{[3]}=-\frac{i \theta^{\alpha \beta}}{4}\left[\hat{A}_{\mu},\left\{\hat{A}_{\alpha}, \partial_{\beta} \hat{C}\right\}\right] .
\end{aligned}
$$

At second and third order in the fields Eq. (B.14) yields:

$$
\hat{\gamma}^{[0]} \eta_{\mu}^{[2]}=0, \quad \hat{\gamma}^{[0]} \eta_{\mu}^{[3]}+\hat{\gamma}^{[1]} \eta_{\mu}^{[2]}+\left[\hat{C}^{*}, \eta_{\mu}^{[2]}\right]=0 .
$$

Again, there exists $\xi_{\mu}^{[2]}$ such that $\eta_{\mu}^{[2]}=\hat{\gamma}^{[0]} \xi_{\mu}^{[2]}$, and a convenient choice turns out to be

$$
\xi_{\mu}^{[2]}=\frac{i \theta^{\alpha \beta}}{4}\left\{\partial_{\alpha} \hat{A}_{\mu}+\hat{F}_{\alpha \mu}^{[0]} * \hat{A}_{\beta}\right\}
$$

Inserting this result in the second equation, the latter reads $\hat{\gamma}^{[0]}\left(\eta_{\mu}^{[3]}-\hat{\gamma}^{[1]} \xi_{\mu}^{[2]}-\left[\hat{C}^{*}, \xi_{\mu}^{[2]}\right]\right)=$ 0 . Again, one concludes that there exists $\xi_{\mu}^{[3]}$ such that

$$
\eta_{\mu}^{[3]}-\hat{\gamma}^{[1]} \xi_{\mu}^{[2]}-\left[\hat{C}^{*}, \xi_{\mu}^{[2]}\right]=\hat{\gamma}^{[0]} \xi_{\mu}^{[3]}
$$

a convenient choice being

$$
\xi_{\mu}^{[3]}=\frac{i \theta^{\alpha \beta}}{4}\left\{\left[\hat{A}_{\alpha}^{*}, \hat{A}_{\mu}\right] * \hat{A}_{\beta}\right\} .
$$


The resultant $\xi_{\mu}^{[3]}$ satisfies $\hat{\gamma}^{[1]} \xi_{\mu}^{[3]}=-\left[\hat{C}^{*}, \xi_{\mu}^{[3]}\right]$ which implies $\hat{\gamma}^{[1]} \int d^{n} x \hat{A}_{A}^{* \mu} \xi_{\mu}^{[3] A}=0$. All in all this yields

$$
\frac{\partial \hat{S}_{1}}{\partial \vartheta}-\hat{\delta} \hat{\Xi}_{2}=\hat{\gamma} \hat{\Xi}_{1}
$$

with $\hat{\Xi}_{1}=-\int d^{n} x \hat{A}_{A}^{* \mu}\left(\xi_{\mu}^{[2]}+\xi_{\mu}^{[3]}\right)^{A}$. Explicitly one obtains

$$
\hat{\Xi}_{1}=-\frac{i \theta^{\alpha \beta}}{4} \int d^{n} x \hat{A}_{A}^{* \mu}\left\{\hat{F}_{\alpha \mu}+\partial_{\alpha} \hat{A}_{\mu}^{*}, \hat{A}_{\beta}\right\}^{A}
$$

Using (B.12) and (B.23) in (B.1), we obtain

$$
\frac{\partial \hat{S}}{\partial \vartheta}=\hat{B}_{0}+\hat{s} \hat{\Xi}
$$

with $\hat{\Xi}=\hat{\Xi}_{1}+\hat{\Xi}_{2}$ and $\hat{B}_{0}=\frac{\partial \hat{S}_{0}}{\partial \vartheta}-\hat{\delta} \hat{\Xi}_{1}$.

We now turn to the question on how to "invert" $\hat{\gamma}^{[0]}$. In the space of fields and their derivatives, we introduce the following new coordinates $\hat{y}^{\alpha}, \hat{z}^{\alpha}, \hat{w}^{i}$ :

$$
\begin{aligned}
& \left\{\hat{y}^{\alpha}\right\}=\left\{\partial_{\left(\nu_{1}\right.} \ldots \partial_{\nu_{l}} \hat{A}_{\mu)}^{A}\right\}, \quad\left\{\hat{z}^{\alpha}\right\}=\left\{\partial_{\left(\nu_{1}\right.} \ldots \partial_{\nu_{l}} \partial_{\mu)} \hat{C}^{A}\right\}, \\
& \left.\left\{\hat{w}^{i}\right\}=\left\{\hat{C}^{A}, \hat{\chi}_{\Delta}^{[0] A}\right\}, \hat{\chi}_{\Delta}^{[0] A} \equiv \partial_{\left(\nu_{1}\right.} \ldots \partial_{\nu_{l}} \hat{F}_{\mu) \lambda}^{[0] A}\right\},
\end{aligned}
$$

where $l=0,1, \ldots$ and $\hat{F}_{\mu \nu}^{[0] A}=\partial_{\mu} \hat{A}_{\nu}^{A}-\partial_{\nu} \hat{A}_{\mu}^{A}$. These variables are independent and complete in the sense that every local function of fields and their derivatives can again be uniquely expressed in terms of them.

We now define the contracting homotopy

$$
\hat{\rho}^{[0]} f(\hat{y}, \hat{z}, \hat{w})=\int_{0}^{1} \frac{d t}{t}\left[\hat{y}^{\alpha} \frac{\partial^{L}}{\partial \hat{z}^{\alpha}} f\right](t \hat{y}, t \hat{z}, \hat{w}),
$$

which satisfies

$$
\left\{\hat{\gamma}^{[0]}, \hat{\rho}^{[0]}\right\} f(\hat{y}, \hat{z}, \hat{w})=f(\hat{y}, \hat{z}, \hat{w})-f(0,0, \hat{w}) .
$$

It follows that any $\hat{\gamma}^{[0]}$-closed function that vanishes when $\hat{y}=\hat{z}=0$, is $\hat{\gamma}^{[0]}$-exact. This is the case for $\eta, \eta_{\mu}^{[2]}$, and $\eta_{\mu}^{[3]}-\hat{\gamma}^{[1]} \xi_{\mu}^{[2]}-\left[\hat{C}^{*}, \xi_{\mu}^{[2]}\right]$ because all of them depend explicitly on $\hat{z}^{\alpha}$. In particular, $\hat{\gamma}^{[0]} \eta=0$ implies $\eta=\hat{\gamma}^{[0]} \xi^{\prime}$ with $\xi^{\prime}=\hat{\rho}^{[0]} \eta$.

However, the expression for $\xi^{\prime}=\hat{\rho}^{[0]} \eta$ is rather complicated because of higher derivatives of $C$ in $\eta$. Because the general solution to $\eta=\hat{\gamma}^{[0]} \tilde{\xi}$ is $\tilde{\xi}=\xi^{\prime}+\nu$ with $\hat{\gamma}^{[0]} \nu=0$, one can use this freedom to arrive at the particular solution $\xi$ given above. A way to get the expressions for $\xi, \xi_{\mu}^{[2]}$, and $\xi_{\mu}^{[3]}$ used in the text is to consider $\hat{\rho}_{*}^{[0]}$ which coincides with $\hat{\rho}^{[0]}$ on the variables $\hat{y}, \hat{z}, \hat{w}$ but satisfies Leibnitz rule on the star polynomials $\eta, \eta_{\mu}^{[2]}$, and $\eta_{\mu}^{[3]}-\hat{\gamma}^{[1]} \xi_{\mu}^{[2]}-\left[\hat{C}^{*}, \xi_{\mu}^{[2]}\right]$ in these variables. 


\section{Appendix C: Proof of proposition 6}

Given arbitrary $\Lambda$ and $r$, together with $W_{\mu}$ satisfying (3.52) and (3.53), it can be directly checked that $f_{\mu}^{c}$ and $h^{c}$ defined by (3.50) and (3.51) satisfy (3.44) and (3.45).

Conversely, given $f_{\mu}^{c}$ and $h^{c}$ satisfying (3.44) and (3.45) and the boundary conditions (3.46) $, \Lambda, r$, and $W_{\mu}$ can be constructed order by order. Taking $\stackrel{0}{r}=\left.h^{c}\right|_{\mathcal{U}}-C$ and $\stackrel{0}{\Lambda}=1$, the equations (3.50) and (3.51) are satisfied at order zero in $\vartheta$. Here $\left.h^{c}\right|_{\mathcal{U}}$ denotes the function obtained by putting to zero all variables except for the undifferentiated ghosts $C^{A}$. Because $\gamma$ commutes with putting to zero these variables, equation (3.44) implies that $\stackrel{0}{r}$ satisfies (3.52). Notice also that $\left(A_{\mu}+W_{\mu}\right)+\left(A_{\mu}^{A}+W_{\mu}^{A}\right) \frac{\partial r}{\partial C^{A}}$ satisfies equation (3.45) for any $\bar{\gamma}$-closed $W_{\mu}$ provided $\stackrel{0}{r}$ satisfies (3.52).

Assume that we have found $\stackrel{k}{\Lambda}, \stackrel{k}{r}$, and $\stackrel{k}{W}$, satisfying (3.52) and (3.53) such that

$$
\begin{aligned}
& h^{c}=\stackrel{k}{\Lambda}^{-1}(C+\stackrel{k}{r}) \stackrel{k}{\Lambda}+\stackrel{k}{\Lambda}^{-1} \gamma \stackrel{k}{\Lambda}+\vartheta^{k+1} \sigma^{(k+1)}+O\left(\vartheta^{k+2}\right) \\
& f^{c}=\stackrel{k}{\Lambda}^{-1}\left(\left(A_{\mu}+\stackrel{k}{W}_{\mu}\right)+\left(A_{\mu}^{A}+\stackrel{k}{W}_{\mu}^{A}\right) \frac{\partial \stackrel{k}{r}}{\partial C^{A}}\right) \stackrel{k}{\Lambda}+\stackrel{k}{\Lambda}{ }^{-1} \gamma \stackrel{k}{\Lambda}+\vartheta^{k+1} \sigma_{\mu}^{(k+1)}+O\left(\vartheta^{k+2}\right)
\end{aligned}
$$

It then follows from equations (3.44) and (3.45) that $\sigma^{(k+1)}$ and $\sigma_{\mu}^{(k+1)}$ satisfy

$$
\bar{\gamma} \sigma^{(k+1)}=0, \quad \bar{\gamma} \sigma_{\mu}^{(k+1)}=D_{\mu} \sigma^{(k+1)} .
$$

The general solution $\sigma^{(k+1)}$ is given by

$$
\sigma^{(k+1)}=\bar{\gamma} \lambda^{(k+1)}+r^{(k+1)}
$$

where $r^{(k+1)}=C^{A}\left(r^{(k+1)}\right){ }_{A}^{B} T_{B}$ satisfies

$$
\bar{\gamma} r^{(k+1)}=0 .
$$

This follows from the covariant Poincaré lemma for generic Lie algebras proved in 33 ] (see also [23, 24] for the reductive case). Indeed, $\sigma^{(k+1)}=j^{(k+1)}(\chi, C)+\bar{\gamma} \mu^{(k+1)}$, where the $\chi$ variables have been defined in (3.27). By considering the terms which depend only on $\chi$ and $C$, the second equation of (C.3) reduces to

$$
\frac{\partial j^{(k+1)}}{\partial x^{\mu}}+D_{\mu} \chi_{\Delta}^{A} \frac{\partial j^{(k+1)}}{\partial \chi_{\Delta}^{A}}+\bar{\gamma} l^{(k+1)}(\chi, C)=0 .
$$

In this equation, consider a decomposition according to the homogeneity in the original fields and assume (without loss of generality) that $j^{(k+1)}$ starts at order M, with $\chi$ replaced by the abelian $\chi^{[0]}$ 's defined in appendix B. To lowest order, (C.6) coincides with the left hand side of eq. (4.57) of [33] (in the particular case of form degree 0 and $\mathrm{k}=1$; the fact that the index is upper instead of lower and that $\bar{\gamma}$ differs from $\gamma_{1}^{R}$ by a sign in the first term plays no role in the following argument.) According to the first paragraph after $(4.57)$, we can deduce that $j_{M}^{(k+1)}=\delta_{M}^{1} r^{(k+1)}+\bar{\gamma} n_{M}^{(k+1)}\left(\chi^{[0]}, C\right)$, where $r^{(k+1)}$ depends only on the undifferentiated ghosts, $r^{(k+1)}=C^{B}\left(r^{(k+1)}\right)_{B}^{A} T_{A}$ with $\left(r^{(k+1)}\right)_{B}^{A}$ 


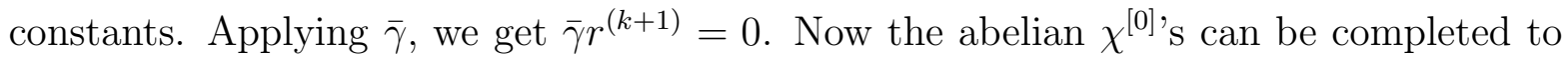
non abelian ones to deduce that $j^{(k+1)}=r^{(k+1)}+\bar{\gamma} n^{(k+1)}(\chi, C)$ with $\bar{\gamma} r^{(k+1)}=0$.

For $\sigma^{(k+1)}$ given by (C.4), the general solution for $\sigma_{\mu}^{(k+1)}$ is

$$
\sigma_{\mu}^{(k+1)}=D_{\mu} \lambda^{(k+1)}+w_{\mu}^{(k+1)}+A_{\mu}^{A} \frac{\partial}{\partial C^{A}} r^{(k+1)},
$$

with $w_{\mu}^{(k+1)}=w_{\mu}^{(k+1)}[A, \vartheta]$ satisfying $\bar{\gamma} w_{\mu}^{(k+1)}=0$.

Taking then

$$
\begin{gathered}
\stackrel{k+1}{\Lambda}=\exp \left(\vartheta^{k+1} \lambda^{(k+1)}\right) \stackrel{k}{\Lambda}, \quad{ }^{k+1}=\left.\left(\stackrel{k+1}{\Lambda} h^{c} \stackrel{k+1}{\Lambda}-C\right)\right|_{\mathcal{U}} \\
{ }^{k+1}{ }_{\mu}=W_{\mu}^{k}+\vartheta^{k+1} w_{\mu}^{k+1}
\end{gathered}
$$

one finds that equations (3.44) and (3.45) hold up to order $k+2$, provided that ${ }^{k+1}-\stackrel{k}{r}$ starts at order $\vartheta^{k+1}$. This is indeed the case as one can see by putting to zero all variables except for the undifferentiated ghosts in equation (C.1) and expressing $\stackrel{k}{r}$ through $\left.h^{c}\right|_{\mathcal{U}}$.

It remains to be shown that $\stackrel{k+1}{r}$ satisfies (3.52). Indeed, by putting to zero all variables except for the undifferentiated ghosts in equation (3.44), one concludes that $\left.h^{c}\right|_{\mathcal{U}}$ satisfies (3.44) and then that $\stackrel{k+1}{r}=\left.\left(\stackrel{k+1}{\Lambda} h^{c} \stackrel{k+1^{-1}}{\Lambda}\right)\right|_{\mathcal{U}}-C$ satisfies (3.52).

\section{Appendix D: Proof of theorem 1}

Combining proposition [5] with proposition 6, the ambiguity in the SW map is expressed as a composition of the "commutative" gauge ambiguity described by $\Lambda$ and the substitutions $C^{A} \rightarrow C^{A}+C^{B} r_{B}^{A}, A_{\mu}^{A} \rightarrow A_{\mu}^{A}+W_{\mu}^{A}+\left(A_{\mu}^{B}+W_{\mu}^{B}\right) r_{B}^{A}$. The only nontrivial point in the proof is to show that the commutative gauge ambiguity is described as a noncommutative gauge ambiguity with some $\hat{\Lambda}$.

Given a particular solution $f_{\mu}, h$ to (3.18) and (3.19) and given

$$
\hat{\Lambda}(t)=P \exp _{*}\left(\int_{0}^{t} d \tau \hat{\lambda}(\tau)\right), \quad \frac{d}{d t} \hat{\Lambda}(t)=\hat{\Lambda}(t) * \hat{\lambda}(t), \quad \hat{\Lambda}(0)=\mathbf{1},
$$

with arbitrary $\hat{\lambda}(t)=\hat{\lambda}^{A}[A ; \vartheta, t] T_{A}$, let us introduce the one parameter family of solutions

$$
\begin{aligned}
h^{\prime}(t) & =\hat{\Lambda}^{-1}(t) * h * \hat{\Lambda}(t)+\hat{\Lambda}^{-1}(t) * \gamma \hat{\Lambda}(t), \\
f_{\mu}^{\prime}(t) & =\hat{\Lambda}^{-1}(t) * f_{\mu} * \hat{\Lambda}(t)+\hat{\Lambda}^{-1}(t) * \partial_{\mu} \hat{\Lambda}(t) .
\end{aligned}
$$

It is the unique solution to the differential equations

$$
\frac{\partial h^{\prime}(t)}{\partial t}=\gamma \hat{\lambda}(t)+\left[h^{\prime}(t), \cdot \hat{\lambda}(t)\right] \quad \frac{\partial f_{\mu}^{\prime}(t)}{\partial t}=\partial_{\mu} \hat{\lambda}(t)+\left[f_{\mu}^{\prime}(t) * \hat{\lambda}(t)\right],
$$

subject to the boundary conditions $h^{\prime}(0)=h$ and $f_{\mu}^{\prime}(0)=f_{\mu}$. Similar equations hold for $f_{\mu}^{c}, h^{c}$ and $f_{\mu}^{c^{\prime}}(t), h^{c^{\prime}}(t)$ with star multiplication replaced by standard multiplication. 
Using (3.43) with $f_{\mu}^{c}, h^{c}$ replaced by $f_{\mu}^{c^{\prime}}(t), h^{c^{\prime}}(t)$, one gets

$$
\begin{aligned}
& \frac{\partial h^{\prime}(t)}{\partial t}=\left.\frac{\partial h}{\partial A_{\mu,(\sigma)}}\right|_{A_{\nu} \rightarrow f_{\nu}^{c^{\prime}}(t), C \rightarrow h^{c^{\prime}}(t)} \partial_{(\sigma)}\left(\partial_{\mu} \lambda+\left[f_{\mu}^{c \prime}(t), \lambda\right]\right)+ \\
& \quad+\left.\frac{\partial h}{\partial C_{,(\sigma)}}\right|_{A_{\nu} \rightarrow f_{\nu}^{c^{\prime}}(t), C \rightarrow h^{c^{\prime}}(t)} \partial_{(\sigma)}\left(\gamma \lambda+\left[h^{c^{\prime}}(t), \lambda\right]\right)= \\
& =\left.\left(\frac{\partial h}{\partial A_{\mu,(\sigma)}} \partial_{(\sigma)}\left(\partial_{\mu} \tilde{\lambda}+\left[A_{\mu}, \tilde{\lambda}\right]\right)+\frac{\partial h}{\partial C_{,(\sigma)}} \partial_{(\sigma)}(\gamma \tilde{\lambda}+[C, \tilde{\lambda}])\right)\right|_{A_{\nu} \rightarrow f_{\nu}^{c^{\prime}}(t), C \rightarrow h^{c^{\prime}}(t)}
\end{aligned}
$$

and

$$
\begin{aligned}
\frac{\partial f_{\mu}^{\prime}(t)}{\partial t}=\left.\frac{\partial f_{\mu}}{\partial A_{\nu,(\sigma)}}\right|_{A_{\rho} \rightarrow f_{\rho}^{c^{\prime}}(t)} \partial_{(\sigma)}\left(\partial_{\nu} \lambda+\right. & {\left.\left[f_{\nu}^{c \prime}(t), \lambda\right]\right)=} \\
& =\left.\left(\frac{\partial f_{\mu}}{\partial A_{\nu,(\sigma)}} \partial_{(\sigma)}\left(\partial_{\nu} \tilde{\lambda}+\left[A_{\nu}, \tilde{\lambda}\right]\right)\right)\right|_{A_{\rho} \rightarrow f_{\rho}^{c^{\prime}(t)}}
\end{aligned}
$$

where $\tilde{\lambda}\left[f^{c^{\prime}}[A ; \vartheta, t], \vartheta\right]=\lambda[A ; \vartheta]$ and the repeated multi-index $(\sigma)$ denotes a summation over the derivatives of the fields as in (3.20). Applying $\tilde{\lambda}_{,(\sigma)} \frac{\partial}{\partial C_{.(\sigma)}}$ to (13.18) and (3.19) and using the result in the right hand sides of the equations (D.4) and (D.5) one arrives at (D.3) with

$$
\hat{\lambda}[A ; \vartheta, t]=\lambda_{,(\sigma)}^{A}\left(\left.\frac{\partial h^{c^{\prime}}}{\partial C_{,(\sigma)}^{A}}\right|_{A_{\mu} \rightarrow f_{\mu}^{c^{\prime}}[A ; \vartheta, t]}\right)
$$

\section{Appendix E: Proof of proposition 7}

For $J_{\mu}^{\nu}=\theta_{\mu \rho} \eta^{\rho \nu}$, equations (4.15) imply

$$
J_{\rho}^{\mu} \lambda_{\nu}^{\rho}-J_{\nu}^{\rho} \lambda_{\rho}^{\mu}=0
$$

We first solve this condition and then describe those $\lambda$ that also preserve $\eta$ (and hence $\theta$ ). Let $\eta_{\mu \nu}$ and $\theta_{\mu \nu}$ be the components of the symmetric, respectively antisymmetric, nondegenerate bilinear forms $\eta$ and $\theta$ on $V$, the space of space-time vectors. The defining relation for $J$ then reads

$$
\theta(a, b)=\eta(J a, b)
$$

Since $J$ cannot in general be diagonalized over $\mathbb{R}$, it is useful to consider first $K=J^{2}$, which is a symmetric operator with respect to $\eta$. Because we have assumed $K$ to be diagonalizable over $\mathbb{R}$, we have

$$
K=\left(\begin{array}{cccc}
K_{1} & 0 & \ldots & 0 \\
0 & K_{2} & \ldots & 0 \\
\ldots & \ldots & \ldots & \ldots \\
0 & 0 & \ldots & K_{N}
\end{array}\right), \quad K_{\alpha}=t_{\alpha} \mathbf{1}_{\alpha}
$$


where $t_{\alpha}$ are different eigenvalues of $K, t_{\alpha} \neq t_{\beta}$ for $\alpha \neq \beta$, no summation over $\alpha$ is implied, and $\mathbf{1}_{\alpha}$ is the $m_{\alpha} \times m_{\alpha}$ unit matrix. We denote by $N$ the number of different eigenvalues of $K$ so that $m_{1}+\ldots+m_{N}=n$.

It follows from $[J, K]=0$ that $J$ has also a block diagonal structure in this basis:

$$
J=\left(\begin{array}{cccc}
J_{1} & 0 & \ldots & 0 \\
0 & J_{2} & \ldots & 0 \\
\ldots & \ldots & \ldots & \ldots \\
0 & 0 & \ldots & J_{N}
\end{array}\right), \quad K_{\alpha}=J_{\alpha}^{2}
$$

Similarly, the bilinear form $\eta$ is also block diagonal, with blocks denoted by $\eta_{\alpha}$.

The condition $[\lambda, J]=[\lambda, K]=0$ now implies that $\lambda$ is also block diagonal, with blocks $\lambda_{\alpha}$. The problem then decomposes into

$$
\left[\lambda_{\alpha}, J_{\alpha}\right]=0, \quad \eta_{\alpha}\left(\lambda_{\alpha} a, b\right)+\eta_{\alpha}\left(a, \lambda_{\alpha} b\right)=0
$$

for any $a, b$ belonging to the eigenspace $V_{\alpha}$ of $K$ with eigenvalue $t_{\alpha}$. If $\mathfrak{g}_{\alpha}$ is the Lie algebra of $\lambda_{\alpha}$ satisfying (E.5), the Lie algebra $\mathfrak{g}$ of solutions to (4.15) is isomorphic to the direct sum

$$
\mathfrak{g} \simeq \mathfrak{g}_{1} \oplus \ldots \oplus \mathfrak{g}_{N}
$$

When analyzing equations (E.5) for a given $\alpha$, one can always assume that $J_{\alpha}^{2}= \pm \mathbf{1}_{\alpha}$.

$J_{\alpha}^{2}=-\mathbf{1}_{\alpha}$ : In this case, the eigenspace has even dimension $2 n_{\alpha}$ and $J_{\alpha}$ can be interpreted as a complex structure. The condition that $\eta_{\alpha}\left(J_{\alpha} a, b\right)=-\eta_{\alpha}\left(a, J_{\alpha} b\right) \forall a, b \in V_{\alpha}$ implies that $\eta_{\alpha}\left(J_{\alpha} a, J_{\alpha} b\right)=\eta_{\alpha}(a, b) \forall a, b \in V_{\alpha}$ which in turn means that $\eta_{\alpha}$ is (pseudo)hermitian. Equations (E.5) are then respectively the conditions that $\lambda_{\alpha}$ is complex linear and that it preserves the hermitian form $\eta_{\alpha}$. Thus $\lambda_{\alpha}$ belongs to the unitary algebra $u\left(n_{\alpha}^{+}, n_{\alpha}^{-}\right), n_{\alpha}^{+}+n_{\alpha}^{-}=n_{\alpha}$, where $\left(n_{\alpha}^{+}, n_{\alpha}^{-}\right)$is the signature of the hermitian form $\eta_{\alpha}$.

$J_{\alpha}^{2}=\mathbf{1}_{\alpha}$ : In this case, it is useful to interpret $J_{\alpha}$ as a so-called "polarization structure". For such a $J_{\alpha}=\mathbf{1}_{\alpha}$, one can always introduce a basis of eigenvectors $e_{i}, \bar{e}_{\bar{i}}$ corresponding to the eigenvalues +1 and -1 , respectively. It then follows from $\eta_{\alpha}\left(J_{\alpha} e_{i}, e_{j}\right)+$ $\eta_{\alpha}\left(e_{i}, J_{\alpha} e_{j}\right)=0$ that $\eta_{\alpha}\left(e_{i}, e_{j}\right)=0$. The same arguments show that $\eta_{\alpha}\left(\bar{e}_{\bar{i}}, \bar{e}_{\bar{j}}\right)=0$. Finally, non-degeneracy of $\eta_{\alpha}$ implies that the number of $e_{i}$ and $\bar{e}_{\bar{i}}$ coincides. In the basis $e, \bar{e}$ it is easy to solve the equations (E.5). Indeed, a general solution to $\left[\lambda_{\alpha}, J_{\alpha}\right]=0$ is given by a block diagonal matrix $\lambda_{\alpha}$ with two arbitrary blocks of dimension $n_{\alpha} \times n_{\alpha}$. At the same time the second equation in (E.5) says that only one of them is independent. Thus the Lie algebra of solutions $\lambda_{\alpha}$ is $g l\left(n_{\alpha}, \mathbb{R}\right)$.

\section{References}

[1] N. Seiberg and E. Witten, "String theory and noncommutative geometry," JHEP 09 (1999) 032, hep-th/9908142. 
[2] G. Barnich and M. Henneaux, "Consistent couplings between fields with a gauge freedom and deformations of the master equation," Phys. Lett. B311 (1993) 123-129, hep-th/9304057

[3] M. Henneaux, "Consistent interactions between gauge fields: The cohomological approach," in Secondary Calculus and Cohomological Physics, A. V. M. Henneaux, J. Krasil'shchik, ed., vol. 219 of Contemporary Mathematics, pp. 93-109. Amercian Mathematical Society, 1997. hep-th/9712226.

[4] C. Becchi, A. Rouet, and R. Stora, "Renormalization of gauge theories," Annals Phys. 98 (1976) 287.

[5] I. V. Tyutin, "Gauge invariance in field theory and statistical physics in operator formalism,". LEBEDEV-75-39.

[6] J. Zinn-Justin, "Renormalization of gauge theories," in Trends in Elementary Particle Physics, H. Rollnik and K. Dietz, eds., Lectures Notes in Physics 37. Springer Verlag, Berlin, 1975.

[7] J. A. Dixon, "Field redefinition and renormalization in gauge theories," Nucl. Phys. B99 (1975) 420.

[8] I. A. Batalin and G. A. Vilkoviskii, "Gauge algebra and quantization," Phys. Lett. 102B (1981) 27.

[9] I. A. Batalin and G. A. Vilkoviskii, "Feynman rules for reducible gauge theories," Phys. Lett. 120B (1983) 166.

[10] I. A. Batalin and G. A. Vilkoviskii, "Quantization of gauge theories with linearly dependent generators," Phys. Rev. D28 (1983) 2567-2582.

[11] I. A. Batalin and G. A. Vilkoviskii, "Closure of the gauge algebra, generalized Lie equations and Feynman rules," Nucl. Phys. B234 (1984) 106.

[12] I. A. Batalin and G. A. Vilkoviskii, "Existence theorem for gauge algebra," J. Math. Phys. 26 (1985) 172.

[13] M. Henneaux and C. Teitelboim, Quantization of Gauge Systems. Princeton University Press, 1992.

[14] J. Gomis, J. París, and S. Samuel, "Antibracket, antifields and gauge theory quantization," Phys. Rept. 259 (1995) 1-145, hep-th/9412228

[15] G. Barnich, M. Grigoriev, and M. Henneaux, "Seiberg-Witten maps from the point of view of consistent deformations of gauge theories," JHEP 10 (2001) 004, hep-th/0106188.

[16] K. Okuyama, "Comments on open Wilson lines and generalized star products," Phys. Lett. B506 (2001) 377-384, hep-th/0101177. 
[17] D. Brace, B. L. Cerchiai, A. F. Pasqua, U. Varadarajan, and B. Zumino, "A cohomological approach to the non-Abelian Seiberg-Witten map," JHEP 06 (2001) 047, hep-th/0105192.

[18] D. Brace, B. L. Cerchiai, and B. Zumino, "Non-Abelian gauge theories on noncommutative spaces," Int. J. Mod. Phys. A17S1 (2002) 205-217, hep-th/0107225

[19] M. Picariello, A. Quadri, and S. P. Sorella, "Chern-Simons in the Seiberg-Witten map for non-commutative Abelian gauge theories in 4d," JHEP 01 (2002) 045, hep-th/0110101.

[20] B. L. Cerchiai, A. F. Pasqua, and B. Zumino, "The Seiberg-Witten map for noncommutative gauge theories," hep-th/0206231.

[21] J. Gomis, K. Kamimura, and T. Mateos, "Gauge and BRST generators for space-time non-commutative U(1) theory," JHEP 03 (2001) 010, hep-th/0009158.

[22] F. Brandt, "Local BRST cohomology in the antifield formalism." unpublished lecture notes, http://home.nexgo.de/fbrandt/lectures.html.

[23] F. Brandt, N. Dragon, and M. Kreuzer, "Completeness and nontriviality of the solutions of the consistency conditions," Nucl. Phys. B332 (1990) 224-249.

[24] M. Dubois-Violette, M. Henneaux, M. Talon, and C.-M. Viallet, "General solution of the consistency equation," Phys. Lett. B289 (1992) 361-367, hep-th/9206106.

[25] T. Asakawa and I. Kishimoto, "Comments on gauge equivalence in noncommutative geometry," JHEP 11 (1999) 024, hep-th/9909139.

[26] A. Bichl et al., "Renormalization of the noncommutative photon self-energy to all orders via seiberg-witten map," JHEP 06 (2001) 013, hep-th/0104097.

[27] G. Barnich, F. Brandt, and M. Henneaux, "Local BRST cohomology in gauge theories," Phys. Rept. 338 (2000) 439-569, hep-th/0002245.

[28] G. Barnich and M. Henneaux, "Renormalization of gauge invariant operators and anomalies in Yang-Mills theory," Phys. Rev. Lett. 72 (1994) 1588-1591, hep-th/9312206.

[29] G. Barnich, F. Brandt, and M. Henneaux, "Local BRST cohomology in the antifield formalism. II. Application to Yang-Mills theory," Commun. Math. Phys. 174 (1995) 93-116, hep-th/9405194.

[30] N. Grandi and G. A. Silva, "Chern-Simons action in noncommutative space," Phys. Lett. B507 (2001) 345-350, hep-th/0010113.

[31] G. Barnich, F. Brandt, and M. Grigoriev, "Seiberg-Witten maps and noncommutative Yang-Mills theories for arbitrary gauge groups," JHEP 08 (2002) 023, hep-th/0206003 
[32] C. G. Torre, "Natural symmetries of the Yang-Mills equations," J. Math. Phys. 36 (1995) 2113-2130, hep-th/9407129.

[33] A. Barkallil, G. Barnich, and C. Schomblond, "Results on the Wess-Zumino consistency condition for arbitrary Lie algebras," J. Math. Phys. 43 (2002) 5987-6015, math-ph/0205047. 